\title{
Optimal Decision Trees on Simplicial Complexes
}

\author{
Jakob Jonsson* \\ Department of Mathematics, KTH, SE-10044 Stockholm, Sweden \\ jakob_jonsson@yahoo.se
}

Submitted: Jun 13, 2003; Accepted: Oct 15, 2004; Published: Jan 7, 2005

Mathematics Subject Classifications: 05E25, 55U10, 06A11

\begin{abstract}
We consider topological aspects of decision trees on simplicial complexes, concentrating on how to use decision trees as a tool in topological combinatorics. By Robin Forman's discrete Morse theory, the number of evasive faces of a given dimension $i$ with respect to a decision tree on a simplicial complex is greater than or equal to the $i$ th reduced Betti number (over any field) of the complex. Under certain favorable circumstances, a simplicial complex admits an "optimal" decision tree such that equality holds for each $i$; we may hence read off the homology directly from the tree. We provide a recursive definition of the class of semi-nonevasive simplicial complexes with this property. A certain generalization turns out to yield the class of semi-collapsible simplicial complexes that admit an optimal discrete Morse function in the analogous sense. In addition, we develop some elementary theory about semi-nonevasive and semi-collapsible complexes. Finally, we provide explicit optimal decision trees for several well-known simplicial complexes.
\end{abstract}

\section{Introduction}

We examine topological properties of decision trees on simplicial complexes, the emphasis being on how one may apply decision trees to problems in topological combinatorics. Our work is to a great extent based on Forman's seminal papers [14, 15].

Let $\Delta$ be an abstract simplicial complex consisting of subsets of a finite set $E$. One may view a decision tree on the pair $(\Delta, E)$ as a deterministic algorithm A that on input a secret set $\sigma \subseteq E$ asks repeated questions of the form "Is the element $x$ contained in $\sigma$ ?" until all questions but one have been asked. $A$ is allowed to be adaptive in the sense that each question may depend on responses to earlier questions. Let $x_{\sigma}$ be the one element that A never queries. $\sigma$ is nonevasive (and A successful) if $\sigma-x_{\sigma}$ and $\sigma+x_{\sigma}$ are either both in $\Delta$ or both outside $\Delta$. Otherwise, $\sigma$ is evasive.

*Research financed by EC's IHRP Programme, within the Research Training Network "Algebraic Combinatorics in Europe," grant HPRN-CT-2001-00272. 
In this paper, we adopt an "intrinsic" approach, meaning that we restrict our attention to the faces in $\Delta$; whether or not a given subset of $E$ outside $\Delta$ is evasive is of no interest to us. We may thus interpret $\mathrm{A}$ as an algorithm that takes as input a secret face $\sigma \in \Delta$ and tries to save a query $x_{\sigma}$ with the property that $\sigma-x_{\sigma}$ and $\sigma+x_{\sigma}$ are both in $\Delta$. Clearly, a face $\sigma$ is evasive if and only if $\sigma+x_{\sigma} \notin \Delta$. Aligning with this intrinsic approach, we will always assume that the underlying set $E$ is exactly the set of 0-cells (vertices) in $\Delta$.

Given a simplicial complex $\Delta$, a natural goal is to find a decision tree with as few evasive faces as possible. In general, there is no decision tree such that all faces are nonevasive. Specifically, if $\Delta$ is not contractible, then such a decision tree cannot exist; Kahn, Saks, and Sturtevant [21] were the first to observe this. More generally, Forman [15] has demonstrated that a decision tree on $\Delta$ gives rise to an acyclic matching on $\Delta$ (corresponding to a discrete Morse function [14]) such that a face is unmatched (critical) if and only if the face is evasive. One defines the matching by pairing $\sigma-x_{\sigma}$ with $\sigma+x_{\sigma}$ for each nonevasive face $\sigma$, where $x_{\sigma}$ is the element not queried for $\sigma$. As a consequence of discrete Morse theory [14], there are at least $\operatorname{dim} \tilde{H}_{i}(\Delta ; \mathbb{F})$ evasive faces in $\Delta$ of dimension $i$ for any given field $\mathbb{F}$.

The goal of this paper is three-fold:

- The first goal is to develop some elementary theory about "optimal" decision trees. For a given field $\mathbb{F}$, a decision tree on a complex $\Delta$ is $\mathbb{F}$-optimal if the number of evasive faces of dimension $i$ is equal to the Betti number $\operatorname{dim} \tilde{H}_{i}(\Delta ; \mathbb{F})$ for each $i$. We give a recursive definition of the class of semi-nonevasive simplicial complexes that admit an $\mathbb{F}$-optimal decision tree. We also generalize the concept of decision trees to allow questions of the form "Is the set $\tau$ a subset of $\sigma$ ?" This turns out to yield an alternative characterization of discrete Morse theory on simplicial complexes. As a consequence, we may characterize $\mathbb{F}$-optimal acyclic matchings - defined in the natural manner - in terms of generalized decision trees. We will refer to complexes admitting $\mathbb{F}$-optimal acyclic matchings as semi-collapsible complexes, aligning with the fact that collapsible complexes are those admitting a perfect acyclic matching. Vertex-decomposable and shellable complexes constitute important examples of semi-nonevasive and semi-collapsible complexes, respectively.

- The second goal is to investigate under what conditions the properties of being seminonevasive and semi-collapsible are preserved under standard operations such as taking the join of two complexes or forming the barycentric subdivision or Alexander dual of a complex. The results and proofs are similar in nature to those Welker [38] provided for nonevasive and collapsible complexes.

- The third goal is to provide a number of examples demonstrating how one may use optimal decision trees to compute the homotopy type of explicit simplicial complexes. We will concentrate on complexes for which the homotopy type is already known. Yet, our decision trees will give new proofs for the homotopy type, and in 
most cases the proofs are not more complicated - sometimes even simpler - than earlier proofs.

Optimal decision trees appeared in the work of Charalambous [11], Forman [15], and Soll [35]. Recently, Hersh [17] developed powerful techniques for optimizing acyclic matchings; see Hersh and Welker [18] for an application. The complexity-theoretic aspect of optimization is considered in the work of Lewiner, Lopes, and Tavares [23, 24, 25]. For more information about the connection between evasiveness and topology, there are several papers $[31,32,22,21,10]$ and surveys $[3,8]$ to consult.

All topological and homological concepts and results in this paper are defined and stated in terms of simplicial complexes. There are potential generalizations of these concepts and results, either in a topological direction - allowing for a more general class of CW complexes - or in a homological direction - allowing for a more general class of chain complexes. For simplicity and clarity, we restrict our attention to simplicial complexes.

For basic definitions and results about decision trees, see Section 1. Fundamental results about optimal decision trees appear in Section 2; see Section 4 for some operations that preserve optimality. In Section 3, we present some useful constructions that we will use in Section 5, where we examine some concrete examples.

Remark. This paper is a revised version of a preprint from 1999 titled "The decision tree method".

\subsection{Basic concepts}

For $n \geq 1$, define $[n]=\{1, \ldots, n\}$. For a set $\sigma$ and an element $x$, write $\sigma+x=\sigma \cup\{x\}$ and $\sigma-x=\sigma \backslash\{x\}$. We let $|\sigma|$ denote the size of $\sigma$.

A (simple) graph $G=(V, E)$ consists of a finite set $V$ of vertices and a set $E \subseteq\left(\begin{array}{l}V \\ 2\end{array}\right)$ of edges in $G$. The edge between $a$ and $b$ is denoted $a b$ or $\{a, b\}$. A (simple and loopless) digraph $D=(V, A)$ consists of a vertex set $V$ and a set $A \subseteq V \times V \backslash\{(v, v): v \in V\}$ of directed edges. The edge $(v, w)$ is directed from $v$ to $w$.

An (abstract) simplicial complex on a finite set $X$ is a family of subsets of $X$ closed under deletion of elements. We refer to the elements in $X$ as 0 -cells. For the purposes of this paper, we adopt the convention that the empty family - the void complex - is a simplicial complex. Members of a simplicial complex $\Sigma$ are called faces. The dimension of a face $\sigma$ is defined as $|\sigma|-1$. The dimension of a nonempty complex $\Sigma$ is the maximal dimension of any face in $\Sigma$. A complex is pure if all maximal faces have the same dimension. For $d \geq-1$, the $d$-simplex is the simplicial complex of all subsets of a set of size $d+1$. Note that the $(-1)$-simplex (not to be confused with the void complex) contains the empty set and nothing else.

A simplicial complex $\Delta$ is obtained from another simplicial complex $\Delta^{\prime}$ via an elementary collapse if $\Delta^{\prime} \backslash \Delta=\{\sigma, \tau\}$ and $\sigma \varsubsetneqq \tau$. This means that $\tau$ is the only face in $\Delta^{\prime}$ properly containing $\sigma$. If $\Delta$ can be obtained from $\Delta^{\prime}$ via a sequence of elementary 
collapses, then $\Delta^{\prime}$ is collapsible to $\Delta$. If $\Delta$ is void or a 0 -simplex $\{\emptyset,\{v\}\}$, then $\Delta^{\prime}$ is collapsible (to a point); see also Section 2.1 .

For a family $\Delta$ of sets and a set $\sigma$, the $\operatorname{link} \operatorname{link}_{\Delta}(\sigma)$ is the family of all $\tau \in \Delta$ such that $\tau \cap \sigma=\emptyset$ and $\tau \cup \sigma \in \Delta$. The deletion $\operatorname{del}_{\Delta}(\sigma)$ is the family of all $\tau \in \Delta$ such that $\tau \cap \sigma=\emptyset$. We define the face-deletion $\operatorname{fdel}_{\Delta}(\sigma)$ as the family of all $\tau \in \Delta$ such that $\sigma \nsubseteq \tau$. The link, deletion, and face-deletion of a simplicial complex are all simplicial complexes. For a family $\Delta$ of sets and disjoint sets $I$ and $E$, define $\Delta(I, E)=\{\sigma$ : $\sigma \cap(E \cup I)=\emptyset, I \cup \sigma \in \Delta\}=\operatorname{link}_{\operatorname{del}_{\Delta}(E)}(I)$. Viewing a graph $G=(V, E)$ as a simplicial complex, we may define the induced subgraph of $G$ on the vertex set $W \subseteq V$ as the graph $G(\emptyset, V \backslash W)=\left(W, E \cap\left(\begin{array}{c}W \\ 2\end{array}\right)\right)$.

The join of two complexes $\Delta$ and $\Gamma$, assumed to be defined on disjoint sets of 0-cells, is the simplicial complex $\Delta * \Gamma=\{\sigma \cup \tau: \sigma \in \Delta, \tau \in \Gamma\}$. Note that $\Delta * \emptyset=\emptyset$ and $\Delta *\{\emptyset\}=\Delta$. The cone of $\Delta$ is the join of $\Delta$ with a 0 -simplex $\{\emptyset,\{v\}\}$. Cones are collapsible.

For a simplicial complex $\Delta$ on a set $X$ of size $n$, the Alexander dual of $\Delta$ with respect to $X$ is the simplicial complex $\Delta_{X}^{*}=\{\sigma \subseteq X: X \backslash \sigma \notin \Delta\}$. It is well-known that

$$
\tilde{H}_{d}(\Delta ; \mathbb{F}) \cong \tilde{H}^{n-d-3}\left(\Delta_{X}^{*} ; \mathbb{F}\right) \cong \tilde{H}_{n-d-3}\left(\Delta_{X}^{*} ; \mathbb{F}\right)
$$

for any field $\mathbb{F}$; see Munkres [28]. Note that the second isomorphism is not true in general for non-fields such as $\mathbb{Z}$.

The order complex $\Delta(P)$ of a partially ordered set (poset) $P=(X, \leq)$ is the simplicial complex of all chains in $P$; a set $A \subseteq X$ belongs to $\Delta(P)$ if and only if $a \leq b$ or $b \leq a$ for all $a, b \in A$. The direct product of two posets $P=\left(X, \leq_{P}\right)$ and $Q=\left(Y, \leq_{Q}\right)$ is the poset $P \times Q=\left(X \times Y, \leq_{P \times Q}\right)$, where $(x, y) \leq_{P \times Q}\left(x^{\prime}, y^{\prime}\right)$ if and only if $x \leq_{P} x^{\prime}$ and $y \leq_{Q} y^{\prime}$. The face poset $P(\Delta)$ of a simplicial complex $\Delta$ is the poset of nonempty faces in $\Delta$ ordered by inclusion. $\operatorname{sd}(\Delta)=\Delta(P(\Delta))$ is the (first) barycentric subdivision of $\Delta$; it is well-known that $\Delta$ and $\operatorname{sd}(\Delta)$ are homeomorphic.

\subsection{Discrete Morse theory}

In this section, we give a brief review of Forman's discrete Morse theory [14]. More elaborate combinatorial interpretations can be found in the work of Chari [12] and Shareshian [33].

Let $X$ be a set and let $\Delta$ be a finite family of finite subsets of $X$. A matching on $\Delta$ is a family $\mathcal{M}$ of pairs $\{\sigma, \tau\}$ with $\sigma, \tau \in \Delta$ such that no set is contained in more than one pair in $\mathcal{M}$. A set $\sigma$ in $\Delta$ is critical or unmatched with respect to $\mathcal{M}$ if $\sigma$ is not contained in any pair in $\mathcal{M}$.

We say that a matching $\mathcal{M}$ on $\Delta$ is an element matching if every pair in $\mathcal{M}$ is of the form $\{\sigma-x, \sigma+x\}$ for some $x \in X$ and $\sigma \subseteq X$. All matchings considered in this paper are element matchings.

Consider an element matching $\mathcal{M}$ on a family $\Delta$. Let $D=D(\Delta, \mathcal{M})$ be the digraph with vertex set $\Delta$ and with a directed edge from $\sigma$ to $\tau$ if and only if either of the following holds: 
1. $\{\sigma, \tau\} \in \mathcal{M}$ and $\tau=\sigma+x$ for some $x \notin \sigma$.

2. $\{\sigma, \tau\} \notin \mathcal{M}$ and $\sigma=\tau+x$ for some $x \notin \tau$.

Thus every edge in $D$ corresponds to an edge in the Hasse diagram of $\Delta$ ordered by set inclusion; edges corresponding to pairs of matched sets are directed from the smaller set to the larger set, whereas the remaining edges are directed the other way around. An element matching $\mathcal{M}$ is an acyclic matching if $D$ is acyclic: If there is a directed path from $\sigma$ to $\tau$ and a directed path from $\tau$ to $\sigma$ in $D$, then $\sigma=\tau$.

Given an acyclic matching $\mathcal{M}$ on a simplicial complex $\Delta \supsetneqq\{\emptyset\}$, we may without loss of generality assume that the empty set $\emptyset$ is contained in some pair in $\mathcal{M}$. Namely, if all 0 -cells are matched with larger faces, then there is a cycle in the digraph $D(\Delta, \mathcal{M})$. In the following results, $\Delta$ is a simplicial complex and $\mathcal{M}$ is an acyclic matching on $\Delta$ such that the empty set is not critical.

Theorem 0.1 (Forman [14]) $\Delta$ is homotopy equivalent to a $C W$ complex with one cell of dimension $p \geq 0$ for each critical face of dimension $p$ in $\Delta$ plus one additional 0 -cell.

Corollary 0.2 If all critical faces have the same dimension $d$, then $\Delta$ is homotopy equivalent to a wedge of $k$ spheres of dimension $d$, where $k$ is the number of critical faces in $\Delta$.

Theorem 0.3 (Forman [14]) If all critical faces are maximal faces in $\Delta$, then $\Delta$ is homotopy equivalent to a wedge of spheres with one sphere of dimension d for each critical face of dimension $d$.

Theorem 0.4 (Forman [14]) Let $\mathbb{F}$ be a field. Then the number of critical faces of dimension $d$ is at least $\operatorname{dim} \tilde{H}_{d}(\Delta ; \mathbb{F})$ for each $d \geq-1$.

Lemma 0.5 Let $\Delta_{0}$ and $\Delta_{1}$ be disjoint families of subsets of a finite set such that $\tau \not \subset \sigma$ if $\sigma \in \Delta_{0}$ and $\tau \in \Delta_{1}$. If $\mathcal{M}_{i}$ is an acyclic matching on $\Delta_{i}$ for $i=0,1$, then $\mathcal{M}_{0} \cup \mathcal{M}_{1}$ is an acyclic matching on $\Delta_{0} \cup \Delta_{1}$.

Proof. This is obvious; there are no arrows directed from $\Delta_{0}$ to $\Delta_{1}$ in the underlying digraph.

\section{Basic properties of decision trees}

We discuss elementary properties of decision trees and introduce the generalized concept of set-decision trees, the generalization being that arbitrary sets rather than single elements are queried. To distinguish between the two notions, we will refer to ordinary decision trees as "element-decision trees". 

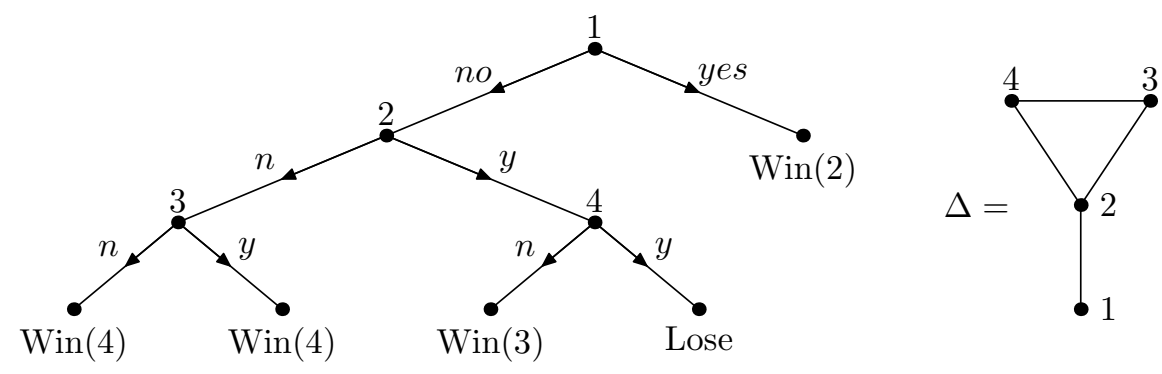

Figure 1: The element-decision tree (1, (2, (3, Win, Win), (4, Win, Lose)), Win) on the complex $\Delta$. "Win $(v)$ " means that the complex corresponding to the given leaf is $\{\emptyset,\{v\}\}$; "Lose" means that the complex is $\{\emptyset\}$.

\subsection{Element-decision trees}

First, we give a recursive definition, suitable for our purposes, of element-decision trees. We are mainly interested in trees on simplicial complexes, but it is convenient to have the concept defined for arbitrary families of sets. Below, the terms "elements" and "sets" always refer to elements and finite subsets of some fixed ground set such as the set of integers.

Definition 1.1 The class of element-decision trees, each associated to a finite family of finite sets, is defined recursively as follows:

(i) $T=$ Win is an element-decision tree on $\emptyset$ and on any 0 -simplex $\{\emptyset,\{v\}\}$.

(ii) $T=$ Lose is an element-decision tree on $\{\emptyset\}$ and on any singleton set $\{\{v\}\}$.

(iii) If $\Delta$ is a family of sets, if $x$ is an element, if $T_{0}$ is an element-decision tree on $\operatorname{del}_{\Delta}(x)$, and if $T_{1}$ is an element-decision tree on $\operatorname{link}_{\Delta}(x)$, then the triple $\left(x, T_{0}, T_{1}\right)$ is an element-decision tree on $\Delta$.

Return to the discussion in the introduction. One may interpret the triple $\left(x, T_{0}, T_{1}\right)$ as follows for a given set $\sigma$ to be examined: The element being queried is $x$. If $x \notin \sigma$, then proceed with $\operatorname{del}_{\Delta}(x)$, the family of sets not containing $x$. Otherwise, proceed with $\operatorname{link}_{\Delta}(x)$, the family with one set $\tau-x$ for each set $\tau$ containing $x$. Proceeding recursively, we finally arrive at a leaf, either Win or Lose. The underlying family being a 0 -simplex $\{\emptyset,\{v\}\}$ means that $\sigma+v \in \Delta$ and $\sigma-v \in \Delta$; we win as $v$ remains to be queried. The family being $\{\emptyset\}$ or $\{\{v\}\}$ means that we cannot tell whether $\sigma \in \Delta$ without querying all elements; we lose.

Note that we allow for the "stupid" decision tree ( $v$, Lose, Lose) on $\{\emptyset,\{v\}\}$; this tree queries the element $v$ while it should not. Also, we allow the element $x$ in (iii) to have the property that no set in $\Delta$ contains $x$, which means that $\operatorname{link}_{\Delta}(x)=\emptyset$, or that all sets in $\Delta$ contain $x$, which means that $\operatorname{del}_{\Delta}(x)=\emptyset$.

A set $\tau \in \Delta$ is nonevasive with respect to an element-decision tree $T$ on $\Delta$ if either of the following holds: 


\section{1. $T=$ Win.}

2. $T=\left(x, T_{0}, T_{1}\right)$ for some $x$ not in $\tau$ and $\tau$ is nonevasive with respect to $T_{0}$.

3. $T=\left(x, T_{0}, T_{1}\right)$ for some $x$ in $\tau$ and $\tau-x$ is nonevasive with respect to $T_{1}$.

This means that $T$ - viewed as an algorithm - ends up on a Win leaf on input $\tau$; use induction. If a set $\tau \in \Delta$ is not nonevasive, then $\tau$ is evasive. For example, the edge 24 is the only evasive face with respect to the element-decision tree in Figure 1. The following simple but powerful theorem is a generalization by Forman [15] of an observation by Kahn, Saks, and Sturtevant [21].

Theorem 1.2 (Forman [15]) Let $\Delta$ be a finite family of finite sets and let $T$ be an element-decision tree on $\Delta$. Then there is an acyclic matching on $\Delta$ such that the critical sets are precisely the evasive sets in $\Delta$ with respect to $T$. In particular, if $\Delta$ is a simplicial complex, then $\Delta$ is homotopy equivalent to a $C W$ complex with exactly one cell of dimension $p$ for each evasive set in $\Delta$ of dimension $p$ and one addition 0 -cell.

Proof. Use induction on the size of $T$. It is easy to check that the theorem holds if $T=$ Win or $T=$ Lose; match $\emptyset$ and $v$ if $\Delta=\{\emptyset, v\}$ and $T=$ Win. Suppose that $T=\left(x, T_{0}, T_{1}\right)$. By induction, there is an acyclic matching on $\operatorname{del}_{\Delta}(x)$ with critical sets exactly those $\sigma$ in $\operatorname{del}_{\Delta}(x)$ that are evasive with respect to $T_{0}$. Also, there is an acyclic matching on $\operatorname{link}_{\Delta}(x)$ with critical sets exactly those $\tau$ in $\operatorname{link}_{\Delta}(x)$ that are evasive with respect to $T_{1}$. Combining these two matchings in the obvious manner, we have a matching with critical sets exactly the evasive sets with respect to $T$; by Lemma 0.5 , the matching is acyclic.

\subsection{Set-decision trees}

We provide a natural generalization of the concept of element-decision trees.

Definition 1.3 The class of set-decision trees, each associated to a finite family of finite sets, is defined recursively as follows:

(i) $T=$ Win is a set-decision tree on $\emptyset$ and on any 0 -simplex $\{\emptyset,\{v\}\}$.

(ii) $T=$ Lose is a set-decision tree on $\{\emptyset\}$ and on any singleton set $\{\{v\}\}$.

(iii) If $\Delta$ is a family of sets, if $\sigma$ is a nonempty set, if $T_{0}$ is a set-decision tree on $\operatorname{fdel}_{\Delta}(\sigma)$, and if $T_{1}$ is a set-decision tree on $\operatorname{link}_{\Delta}(\sigma)$, then the triple $\left(\sigma, T_{0}, T_{1}\right)$ is a set-decision tree on $\Delta$.

A simple example is provided in Figure 2. A set $\tau \in \Delta$ is nonevasive with respect to a set-decision tree $T$ on $\Delta$ if either of the following holds:

1. $T=$ Win.

2. $T=\left(\sigma, T_{0}, T_{1}\right)$ for some $\sigma \nsubseteq \tau$ and $\tau$ is nonevasive with respect to $T_{0}$. 


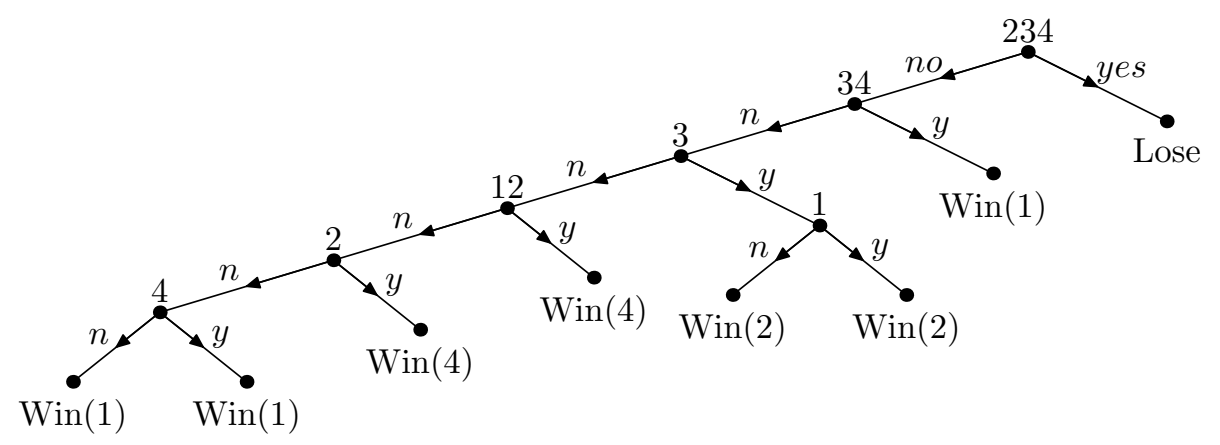

Figure 2: A set-decision tree on the simplicial complex with maximal faces 123, 124, 134, and 234 .

3. $T=\left(\sigma, T_{0}, T_{1}\right)$ for some $\sigma \subseteq \tau$ and $\tau \backslash \sigma$ is nonevasive with respect to $T_{1}$.

If a set $\tau \in \Delta$ is not nonevasive, then $\tau$ is evasive.

Theorem 1.4 Let $\Delta$ be a finite family of finite sets and let $T$ be a set-decision tree on $\Delta$. Then there is an acyclic matching on $\Delta$ such that the critical sets are precisely the evasive sets in $\Delta$ with respect to $T$. Conversely, given an acyclic matching $\mathcal{M}$ on $\Delta$, there is a set-decision tree $T$ on $\Delta$ such that the evasive sets are precisely the critical sets with respect to $\mathcal{M}$.

Proof. For the first part, the proof is identical to the proof of Theorem 1.2. For the second part, first consider the case that $\Delta$ is a complex as in $(i)$ or $(i i)$ in Definition 1.3. If $\Delta=\emptyset$, then $T=$ Win is a set-decision tree with the desired properties, whereas $T=$ Lose is the desired tree if $\Delta=\{\emptyset\}$ or $\Delta=\{\{v\}\}$. For $\Delta=\{\emptyset,\{v\}\}, T=$ Win does the trick if $\emptyset$ and $\{v\}$ are matched, whereas $T=(v$, Lose, Lose) is the tree we are looking for if $\emptyset$ and $\{v\}$ are not matched.

Now, assume that $\Delta$ is some other family. Pick an arbitrary set $\rho \in \Delta$ of maximal size and go backwards in the digraph $D$ of the matching $\mathcal{M}$ until a source $\sigma$ in $D$ is found; there are no edges directed to $\sigma$. Such a $\sigma$ exists as $D$ is acyclic. It is obvious that $|\rho|-1 \leq|\sigma| \leq|\rho|$; in any directed path in $D$, a step up is always followed by and preceded by a step down (unless the step is the first or the last in the path). In particular, $\sigma$ is adjacent in $D$ to any set $\tau$ containing $\sigma$. Since $\sigma$ is matched with at most one such $\tau$ and since $\sigma$ is a source in $D$, there is at most one set containing $\sigma$.

First, suppose that $\sigma$ is contained in a set $\tau$ and hence matched with $\tau$ in $\mathcal{M}$. By induction, there is a set-decision tree $T_{0}$ on $\operatorname{fdel}_{\Delta}(\sigma)=\Delta \backslash\{\sigma, \tau\}$ with evasive sets exactly the critical sets with respect to the restriction of $\mathcal{M}$ to $\operatorname{fdel}_{\Delta}(\sigma)$. Moreover, $\operatorname{link}_{\Delta}(\sigma)=\{\emptyset, \tau \backslash \sigma\}$. Since $T_{1}=$ Win is a set-decision tree on $\operatorname{link}_{\Delta}(\sigma)$ with no evasive sets, it follows that $\left(\sigma, T_{0}, T_{1}\right)$ is a tree with the desired properties. Next, suppose that $\sigma$ is maximal in $\Delta$ and hence critical. By induction, there is a set-decision tree $T_{0}$ on fdel $_{\Delta}(\sigma)=\Delta \backslash\{\sigma\}$ with evasive sets exactly the critical sets with respect to the restriction of $\mathcal{M}$ to $\operatorname{fdel}_{\Delta}(\sigma)$. Moreover, $\operatorname{link}_{\Delta}(\sigma)=\{\emptyset\}$; since $T_{1}=$ Lose is a set-decision tree on $\operatorname{link}_{\Delta}(\sigma)$ with one evasive set, $\left(\sigma, T_{0}, T_{1}\right)$ is a tree with the desired properties. 


\section{Hierarchy of nearly nonevasive complexes}

The purpose of this section is to introduce two families of complexes related to the concept of decision trees:

- Semi-nonevasive complexes admit an element-decision tree with evasive faces enumerated by the reduced Betti numbers over a given field.

- Semi-collapsible complexes admit a set-decision tree with evasive faces enumerated by the reduced Betti numbers over a given field. Equivalently, such complexes admit an acyclic matching with critical faces enumerated by reduced Betti numbers.

One may view these families as generalizations of the well-known families of nonevasive and collapsible complexes:

- Nonevasive complexes admit an element-decision tree with no evasive faces.

- Collapsible complexes admit a set-decision tree with no evasive faces. Equivalently, such complexes admit a perfect acyclic matching.

In Section 2.3, we discuss how all these classes relate to well-known properties such as being shellable and vertex-decomposable. The main conclusion is that the families of seminonevasive and semi-collapsible complexes contain the families of vertex-decomposable and shellable complexes, respectively.

Remark. One may characterize semi-collapsible complexes as follows. Given an acyclic matching on a simplicial complex $\Delta$, we may order the critical faces as $\sigma_{1}, \ldots, \sigma_{n}$ and form a sequence $\emptyset=\Delta_{0} \subset \Delta_{1} \subset \ldots \subset \Delta_{n-1} \subset \Delta_{n} \subseteq \Delta$ of simplicial complexes such that the following is achieved: $\Delta$ is collapsible to $\Delta_{n}, \sigma_{i}$ is a maximal face in $\Delta_{i}$, and $\Delta_{i} \backslash\left\{\sigma_{i}\right\}$ is collapsible to $\Delta_{i-1}$ for $i \in[n]$; compare to the induction proof of Theorem 1.4 (see also Forman [14, Th. 3.3-3.4]). A matching being optimal means that $\sigma_{i}$ is contained in a nonvanishing cycle in the homology of $\Delta_{i}$ for each $i \in[n]$; otherwise the removal of $\sigma_{i}$ would introduce new homology, rather than kill existing homology. With an "elementary semi-collapse" defined either as an ordinary elementary collapse or as the removal of a maximal face contained in a cycle, semi-collapsible complexes are exactly those complexes that can be transformed into the void complex via a sequence of elementary semi-collapses.

\subsection{Nonevasive and collapsible complexes}

It is well-known and easy to see that one may characterize nonevasive and collapsible complexes recursively in the following manner:

Definition 2.1 We define the class of nonevasive simplicial complexes recursively as follows:

(i) The void complex $\emptyset$ and any 0 -simplex $\{\emptyset,\{v\}\}$ are nonevasive. 
(ii) If $\Delta$ contains a 0 -cell $x$ such that $\operatorname{del}_{\Delta}(x)$ and $\operatorname{link}_{\Delta}(x)$ are nonevasive, then $\Delta$ is nonevasive.

Definition 2.2 We define the class of collapsible simplicial complexes recursively as follows:

(i) The void complex $\emptyset$ and any 0 -simplex $\{\emptyset,\{v\}\}$ are collapsible.

(ii) If $\Delta$ contains a nonempty face $\sigma$ such that the face-deletion $\operatorname{fdel}_{\Delta}(\sigma)$ and $\operatorname{link}_{\Delta}(\sigma)$ are collapsible, then $\Delta$ is collapsible.

Clearly, nonevasive complexes are collapsible; this was first observed by Kahn, Saks, and Sturtevant [21]. The converse is not true in general; see Proposition 2.13 in Section 2.3. It is also clear that all cones are nonevasive.

\subsection{Semi-nonevasive and semi-collapsible complexes}

Let $\mathbb{F}$ be a field or $\mathbb{Z}$. A set-decision tree (equivalently, an acyclic matching) on a simplicial complex $\Delta$ is $\mathbb{F}$-optimal if, for each integer $i, \operatorname{dim} \tilde{H}_{i}(\Delta ; \mathbb{F})$ is the number of evasive (critical) faces of dimension $i$; $\operatorname{dim} \tilde{H}_{i}(\Delta ; \mathbb{Z})$ is the rank of the torsion-free part of $\tilde{H}_{i}(\Delta ; \mathbb{Z})$. We define $\mathbb{F}$-optimal element-decision trees analogously. In this section, we define the classes of simplicial complexes that admit $\mathbb{F}$-optimal element-decision or set-decision trees. Our approach is similar to that of Charalambous [11]. See Forman [15] and Soll [35] for more discussion on optimal decision trees.

Definition 2.3 We define the class of semi-nonevasive simplicial complexes over $\mathbb{F}$ recursively as follows:

(i) The void complex $\emptyset$, the (-1)-simplex $\{\emptyset\}$, and any 0 -simplex $\{\emptyset,\{v\}\}$ are seminonevasive over $\mathbb{F}$.

(ii) Suppose $\Delta$ contains a 0-cell $x$ - a shedding vertex (notation borrowed from Provan and Billera [30]) - such that $\operatorname{del}_{\Delta}(x)$ and $\operatorname{link}_{\Delta}(x)$ are semi-nonevasive over $\mathbb{F}$ and such that

$$
\tilde{H}_{d}(\Delta ; \mathbb{F}) \cong \tilde{H}_{d}\left(\operatorname{del}_{\Delta}(x) ; \mathbb{F}\right) \oplus \tilde{H}_{d-1}\left(\operatorname{link}_{\Delta}(x) ; \mathbb{F}\right)
$$

for each $d$. Then $\Delta$ is semi-nonevasive over $\mathbb{F}$.

Definition 2.4 We define the class of semi-collapsible simplicial complexes over $\mathbb{F}$ recursively as follows:

(i) The void complex $\emptyset$, the (-1)-simplex $\{\emptyset\}$, and any 0 -simplex $\{\emptyset,\{v\}\}$ are semicollapsible over $\mathbb{F}$. 
(ii) Suppose that $\Delta$ contains a nonempty face $\sigma$ - a shedding face - such that fdel $\operatorname{s}_{\Delta}(\sigma)$ and $\operatorname{link}_{\Delta}(\sigma)$ are semi-collapsible over $\mathbb{F}$ and such that

$$
\tilde{H}_{d}(\Delta ; \mathbb{F}) \cong \tilde{H}_{d}\left(\operatorname{fdel}_{\Delta}(\sigma) ; \mathbb{F}\right) \oplus \tilde{H}_{d-|\sigma|}\left(\operatorname{link}_{\Delta}(\sigma) ; \mathbb{F}\right)
$$

for each $d$. Then $\Delta$ is semi-collapsible over $\mathbb{F}$.

Clearly, a semi-nonevasive complex over $\mathbb{F}$ is also semi-collapsible over $\mathbb{F}$.

Remark. Let us discuss the identity (3); the discussion also applies to the special case (2). Let $\Delta_{0}=\operatorname{fdel}_{\Delta}(\sigma)$. Note that the relative homology group $\tilde{H}_{d}\left(\Delta, \Delta_{0}\right)=\tilde{H}_{d}\left(\Delta, \Delta_{0} ; \mathbb{F}\right)$ is isomorphic to $\tilde{H}_{d-|\sigma|}\left(\operatorname{link}_{\Delta}(\sigma)\right)$ for each $d$. By the long exact sequence

$$
\ldots \longrightarrow \tilde{H}_{d}\left(\Delta_{0}\right) \longrightarrow \tilde{H}_{d}(\Delta) \longrightarrow \tilde{H}_{d}\left(\Delta, \Delta_{0}\right) \longrightarrow \tilde{H}_{d-1}\left(\Delta_{0}\right) \longrightarrow \ldots
$$

for the pair $\left(\Delta, \Delta_{0}\right),(3)$ is equivalent to the induced map $\partial_{d}^{*}: \tilde{H}_{d}\left(\Delta, \Delta_{0}\right) \longrightarrow \tilde{H}_{d-1}\left(\Delta_{0}\right)$ being zero for each $d$, where $\partial_{d}(z)$ is computed in $\tilde{C}(\Delta)$. This is the case if and only if for every cycle $z \in \tilde{C}\left(\Delta, \Delta_{0}\right)$, there is a $c \in \tilde{C}\left(\Delta_{0}\right)$ with the same boundary as $z$ in $\tilde{C}(\Delta)$. As an important special case, we have the following observation:

Proposition 2.5 If $\tilde{H}_{d}\left(\operatorname{fdel}_{\Delta}(\sigma) ; \mathbb{F}\right)=0$ whenever $\tilde{H}_{d-|\sigma|+1}\left(\operatorname{link}_{\Delta}(\sigma) ; \mathbb{F}\right) \neq 0$, then (3) holds. Hence if $\tilde{H}_{d}\left(\operatorname{del}_{\Delta}(x) ; \mathbb{F}\right)=0$ whenever $\tilde{H}_{d}\left(\operatorname{link}_{\Delta}(x) ; \mathbb{F}\right) \neq 0$, then (2) holds.

The main result of this section is as follows; we postpone the case $\mathbb{F}=\mathbb{Z}$ until the end of the section.

Theorem 2.6 Let $\mathbb{F}$ be a field. A complex $\Delta$ is semi-collapsible over $\mathbb{F}$ if and only if $\Delta$ admits an $\mathbb{F}$-optimal set-decision tree (equivalently, an $\mathbb{F}$-optimal acyclic matching). $\Delta$ is semi-nonevasive over $\mathbb{F}$ if and only if $\Delta$ admits an $\mathbb{F}$-optimal element-decision tree.

Proof. First, we show that every semi-collapsible complex $\Delta$ over $\mathbb{F}$ admits an $\mathbb{F}$-optimal set-decision tree. This is clear if $\Delta$ is as in $(i)$ in Definition 2.4. Use induction and consider a complex derived as in $(i i)$ in Definition 2.4. By induction, $\operatorname{fdel}_{\Delta}(\sigma)$ and $\operatorname{link}_{\Delta}(\sigma)$ admit $\mathbb{F}$-optimal set-decision trees $T_{0}$ and $T_{1}$, respectively. Combining these two trees, we obtain a set-decision tree $T=\left(\sigma, T_{0}, T_{1}\right)$ on $\Delta$. (3) immediately yields that the evasive faces in $\Delta$ are enumerated by the Betti numbers of $\Delta$, and we are done.

Next, suppose that we have an $\mathbb{F}$-optimal set-decision tree $T=\left(\sigma, T_{0}, T_{1}\right) ; T_{0}$ is a tree on $\operatorname{fdel}_{\Delta}(\sigma)$, whereas $T_{1}$ is a tree on $\operatorname{link}_{\Delta}(\sigma)$. We have that $\operatorname{dim} \tilde{H}_{d}(\Delta)=e_{d}$, where $e_{d}$ is the number of evasive faces of dimension $d$ with respect to $T$. Let $a_{d}$ and $b_{d}$ be the number of evasive faces of dimension $d$ with respect to the set-decision trees $T_{0}$ and $T_{1}$, respectively; clearly, $e_{d}=a_{d}+b_{d-|\sigma|}$. By Theorem 0.4, we must have $a_{d} \geq \operatorname{dim} \tilde{H}_{d}\left(\operatorname{fdel}_{\Delta}(\sigma)\right)$ and $b_{d-|\sigma|} \geq \operatorname{dim} \tilde{H}_{d-|\sigma|}\left(\operatorname{link}_{\Delta}(\sigma)\right)$. We want to prove that equality holds for both $a_{d}$ and $b_{d-|\sigma|}$. Namely, this will imply (3) and yield that $T_{0}$ and $T_{1}$ are $\mathbb{F}$-optimal set-decision trees; by induction, we will obtain that each of $\operatorname{fdel}_{\Delta}(\sigma)$ and $\operatorname{link}_{\Delta}(\sigma)$ is semi-collapsible 


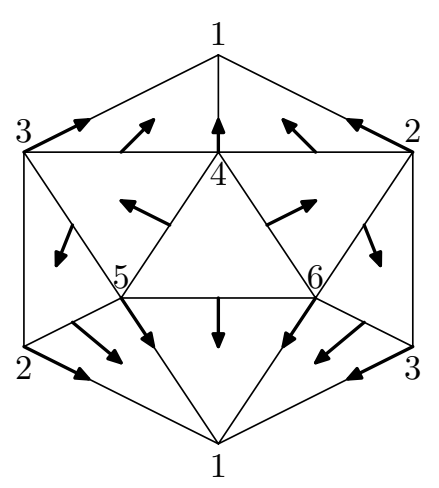

Figure 3: An acyclic matching on a triangulated projective plane with critical faces 23 and 456; 1 is matched with $\emptyset$. This matching is $\mathbb{Z}_{2}$-optimal but not $\mathbb{Q}$-optimal.

and hence that $\Delta$ is semi-collapsible. Now, the long exact sequence (4) immediately yields that

$$
e_{d}=\operatorname{dim} \tilde{H}_{d}(\Delta) \leq \operatorname{dim} \tilde{H}_{d}\left(\operatorname{fdel}_{\Delta}(\sigma)\right)+\operatorname{dim} \tilde{H}_{d-|\sigma|}\left(\operatorname{link}_{\Delta}(\sigma)\right) .
$$

Since the right-hand side is bounded by $a_{d}+b_{d-|\sigma|}=e_{d}$, the inequality must be an equality; thus (3) holds, and we are done.

The last statement in the theorem is proved in exactly the same manner.

Proposition 2.7 If a simplicial complex $\Delta$ is semi-collapsible over $\mathbb{Q}$, then the $\mathbb{Z}$-homology of $\Delta$ is torsion-free. In particular, $\tilde{H}_{d}(\Delta ; \mathbb{Z})=\mathbb{Z}^{\beta_{d}}$, where $\beta_{d}=\operatorname{dim} \tilde{H}_{d}(\Delta ; \mathbb{Q})$. Hence semi-nonevasive complexes over $\mathbb{Q}$ have torsion-free $\mathbb{Z}$-homology.

Proof. This is obvious if $(i)$ in Definition 2.4 holds. Suppose (ii) holds. By induction, the proposition is true for $\operatorname{fdel}_{\Delta}(\sigma)$ and $\operatorname{link}_{\Delta}(\sigma)$. By the remark after Definition 2.4, for every cycle $z \in \tilde{C}\left(\Delta, \operatorname{fdel}_{\Delta}(\sigma) ; \mathbb{Q}\right)$, there is a $c \in \tilde{C}\left(\operatorname{fdel}_{\Delta}(\sigma) ; \mathbb{Q}\right)$ with the same boundary as $z$ in $\tilde{C}(\Delta ; \mathbb{Q})$. As a consequence, for every cycle $z \in \tilde{C}\left(\Delta, \operatorname{fdel}_{\Delta}(\sigma) ; \mathbb{Z}\right)$, there is a $c \in \tilde{C}\left(\operatorname{fdel}_{\Delta}(\sigma) ; \mathbb{Z}\right)$ and an integer $\lambda$ such that $\partial(c)=\lambda \partial(z)$ (computed in $\tilde{C}(\Delta ; \mathbb{Z})$ ). However, since $\mathrm{fdel}_{\Delta}(\sigma)$ is torsion-free, $\lambda \partial(z)$ is a boundary in $\tilde{C}\left(\mathrm{fdel}_{\Delta}(\sigma) ; \mathbb{Z}\right)$ if and only if $\partial(z)$ is a boundary, which implies that there exists a $c^{\prime} \in \tilde{C}\left(\operatorname{fdel}_{\Delta}(\sigma) ; \mathbb{Z}\right)$ such that $\partial\left(c^{\prime}\right)=\partial(z)$. It follows that $\partial_{d}^{*}: \tilde{H}_{d}\left(\Delta, \operatorname{fdel}_{\Delta}(\sigma) ; \mathbb{Z}\right) \longrightarrow \tilde{H}_{d-|\sigma|}\left(\operatorname{fdel}_{\Delta}(\sigma) ; \mathbb{Z}\right)$ is the zero map. Hence (3) holds for $\mathbb{F}=\mathbb{Z}$, and we are done.

Corollary 2.8 A simplicial complex $\Delta$ is semi-collapsible (semi-nonevasive) over $\mathbb{Q}$ if and only if $\Delta$ is semi-collapsible (semi-nonevasive) over $\mathbb{Z}$. If this is the case, then $\Delta$ is semi-collapsible (semi-nonevasive) over every field.

Remark. While the universal coefficient theorem implies that Proposition 2.7 is true for any field of characteristic 0 , the proposition does not remain true for coefficient fields of nonzero characteristic. For example, the triangulated projective plane $\mathbb{R P}^{2}$ in Figure 3 is not semi-collapsible over $\mathbb{Q}$, as the homology has torsion. However, the given acyclic 
matching is $\mathbb{Z}_{2}$-optimal; $\tilde{H}_{1}\left(\mathbb{R P}^{2} ; \mathbb{Z}_{2}\right)=\tilde{H}_{2}\left(\mathbb{R} \mathbb{P}^{2} ; \mathbb{Z}_{2}\right)=\mathbb{Z}_{2}$. In fact, the acyclic matching corresponds to a $\mathbb{Z}_{2}$-optimal element-decision tree in which we first use 4,5 , and 6 as shedding vertices; thus the complex is semi-nonevasive over $\mathbb{Z}_{2}$. A semi-nonevasive complex over $\mathbb{Z}_{3}$ with 3 -torsion is provided in Theorem 5.6.

\subsection{Relations between certain classes of simplicial complexes}

We show how semi-collapsible and semi-nonevasive complexes over $\mathbb{Z}$ relate to vertexdecomposable, shellable, and constructible complexes.

Definition 2.9 We define the class of semipure vertex-decomposable simplicial complexes recursively as follows:

(i) Every simplex (including $\emptyset$ and $\{\emptyset\}$ ) is semipure vertex-decomposable.

(ii) If $\Delta$ contains a 0 -cell $x$ - a shedding vertex - such that $\operatorname{del}_{\Delta}(x)$ and $\operatorname{link}_{\Delta}(x)$ are semipure vertex-decomposable and such that every maximal face in $\operatorname{del}_{\Delta}(x)$ is a maximal face in $\Delta$, then $\Delta$ is also semipure vertex-decomposable.

One may refer to semipure vertex-decomposable complexes that are not pure as nonpure vertex-decomposable. Pure vertex-decomposable complexes were introduced by Provan and Billera [30]. Björner and Wachs [7] extended the concept to nonpure complexes.

Definition 2.10 We define the class of semipure shellable simplicial complexes recursively as follows:

(i) Every simplex (including $\emptyset$ and $\{\emptyset\}$ ) is semipure shellable.

(ii) If $\Delta$ contains a nonempty face $\sigma$ - a shedding face - such that $\operatorname{fdel}_{\Delta}(\sigma)$ and $\operatorname{link}_{\Delta}(\sigma)$ are semipure shellable and such that every maximal face in $\operatorname{fdel}_{\Delta}(\sigma)$ is a maximal face in $\Delta$, then $\Delta$ is also semipure shellable.

One may refer to semipure shellable complexes that are not pure as nonpure shellable. Again, the extension to nonpure complexes is due to Björner and Wachs [6]. To see that Definition 2.10 is equivalent to the original definition [6, Def. 2.1], adapt the proof of Björner and Wachs [7, Th. 11.3].

Chari $[12]$ proved that shellable complexes are semi-collapsible over $\mathbb{Z}$. Let us extend his result to semipure shellable complexes.

Proposition 2.11 Let $\Delta$ be a semipure shellable complex. Then $\Delta$ admits an acyclic matching in which all unmatched faces are maximal faces in $\Delta$. In particular, any semipure shellable complex is semi-collapsible over $\mathbb{Z}$. 
Proof. The proposition is clearly true if $(i)$ in Definition 2.10 is satisfied. Suppose (ii) is satisfied. By induction, $\operatorname{fdel}_{\Delta}(\sigma)$ and $\operatorname{link}_{\Delta}(\sigma)$ admit acyclic matchings such that all unmatched faces are maximal faces. Combining these matchings, we obtain an acyclic matching on $\Delta$. Since maximal faces in $\operatorname{fdel}_{\Delta}(\sigma)$ are maximal faces in $\Delta$, the desired result follows. By Theorem 0.3, $\Delta$ is homotopy equivalent to a wedge of spheres with one sphere of dimension $\operatorname{dim} \sigma$ for each unmatched face $\sigma$; hence $\Delta$ is semi-collapsible.

Soll [35] proved the following result in the pure case.

Proposition 2.12 Semipure vertex-decomposable complexes are semi-nonevasive over $\mathbb{Z}$.

Proof. Use exactly the same approach as in the proof of Proposition 2.11.

Proposition 2.13 Not all shellable complexes are semi-nonevasive.

Proof. The complex with maximal faces 012, 023, 034, 045, 051, 123, 234, 345, 451, 512 is well-known to be shellable and collapsible but not nonevasive or vertex-decomposable. This complex is originally due to Björner (personal communication); see Moriyama and Takeuchi [27, Ex. V6F10-6] and Soll [35, Ex. 5.5.5].

Definition 2.14 We define the class of constructible simplicial complexes recursively as follows:

(i) Every simplex (including $\emptyset$ and $\{\emptyset\}$ ) is constructible.

(ii) If $\Delta_{1}$ and $\Delta_{2}$ are constructible complexes of dimension $d$ and $\Delta_{1} \cap \Delta_{2}$ is a constructible complex of dimension $d-1$, then $\Delta_{1} \cup \Delta_{2}$ is constructible.

Constructible complexes were introduced by Hochster [19]. Every pure shellable complex is constructible, but the converse is not always true; see Björner [3].

Proposition 2.15 Not all constructible complexes are semi-collapsible. Yet, there exist constructible complexes that are nonevasive but not shellable.

Proof. For the first statement, Hachimori [16] has found a two-dimensional contractible and constructible complex without boundary; a complex with no boundary cannot be collapsible. For the second statement, a cone over a constructible complex is constructible and nonevasive but not shellable unless the original complex is shellable.

The results in this section combined with earlier results (see Björner [3]) yield the diagram in Figure 4 of strict implications; "torsion-free" refers to the $\mathbb{Z}$-homology. We refer to Stanley [36] for more information about Cohen-Macaulay $(C M)$ and sequentially CohenMacaulay complexes. Two properties being incomparable in the diagram means that neither of the properties implies the other. We list the nontrivial cases: 


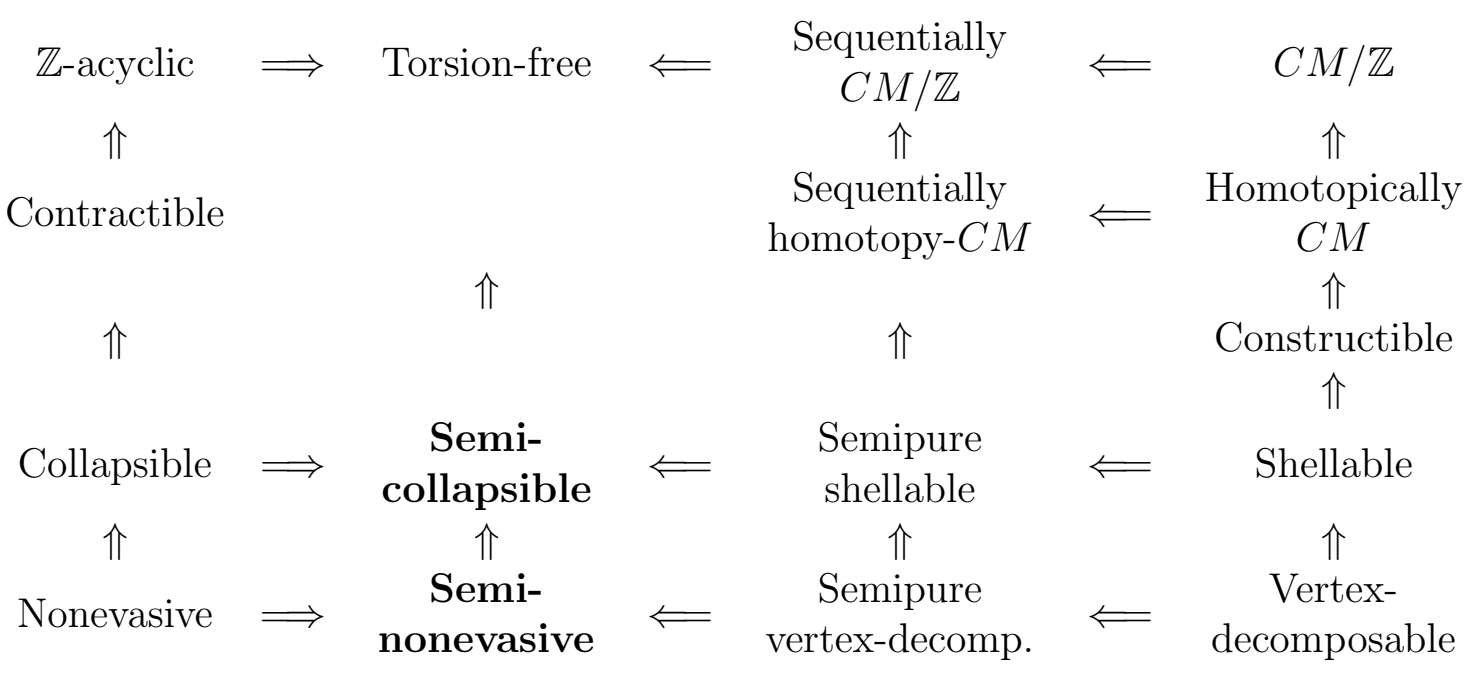

Figure 4: Implications between different classes of simplicial complexes.

- Collapsible or shellable complexes are not necessarily semi-nonevasive. This is Proposition 2.13.

- Contractible or constructible complexes are not necessarily semi-collapsible. This is Proposition 2.15.

\section{Some useful constructions}

Before proceeding, let us introduce some simple but useful constructions that will be used frequently in later sections. For a family $\Delta$ of sets, write $\Delta \sim \sum_{i \geq-1} a_{i} t^{i}$ if there is an element-decision tree on $\Delta$ with exactly $a_{i}$ evasive sets of dimension $i$ for each $i \geq-1$. This notation has the following basic properties; recall from Section 0.1 that $\Delta(I, E)=\operatorname{link}_{\operatorname{del}_{\Delta}(E)}(I):$

Lemma 3.1 Let $\Delta$ be a finite family of finite sets. Then the following hold:

(1) $\Delta$ is nonevasive if and only if $\Delta \sim 0$.

(2) Assume that $\Delta$ is a simplicial complex and let $\mathbb{F}$ be a field. Then $\Delta$ is semi-nonevasive over $\mathbb{F}$ if and only if $\Delta \sim \sum_{i \geq-1} \operatorname{dim} \tilde{H}_{i}(\Delta ; \mathbb{F}) t^{i} ; \Delta$ is semi-nonevasive over $\mathbb{Z}$ if and only if $\Delta \sim \sum_{i \geq-1} \operatorname{dim} \tilde{H}_{i}(\Delta ; \mathbb{Q}) t^{i}$.

(3) Let $v$ be a 0-cell. If $\operatorname{del}_{\Delta}(v) \sim f_{\emptyset}(t)$ and $\operatorname{link}_{\Delta}(v) \sim f_{v}(t)$, then $\Delta \sim f_{\emptyset}(t)+f_{v}(t) t$.

(4) Let $B$ be a set of 0-cells. If $\Delta(A, B \backslash A) \sim f_{A}(t)$ for each $A \subseteq B$, then $\Delta \sim$ $\sum_{A \subseteq B} f_{A}(t) t^{|A|}$. 
(5) Assume that $\Delta$ is a simplicial complex such that $\Delta \sim c t^{d}$. Then $\Delta$ is semi-nonevasive and homotopy equivalent to a wedge of c spheres of dimension d.

Proof. (1) is obvious. To prove (2), use Theorem 2.6 and Corollary 2.8. (3) is obvious, whereas (4) follows from (3) by induction on $|B|$. Finally, by Theorem 1.4, (5) is a consequence of Corollary 0.2.

One may give analogous definitions and results for semi-collapsible complexes, but we will not need them.

Definition 3.2 Let $\Delta$ be a finite family of finite sets. Let $W=\left(w_{1}, \ldots, w_{m}\right)$ be a sequence of distinct elements. The first-hit decomposition of $\Delta$ with respect to $W$ is the sequence consisting of the families $\Delta\left(w_{j},\left\{w_{1}, \ldots, w_{j-1}\right\}\right)$ for $j \in[m]$ and the family $\Delta\left(\emptyset,\left\{w_{1}, \ldots, w_{m}\right\}\right)$.

The term "first-hit" refers to the natural interpretation of the concept in terms of decision trees; for a given set to be checked, query elements in the sequence until some element from the set is found (a first hit).

Lemma 3.3 Let $\Delta$ be a finite family of finite sets and consider the first-hit decomposition of $\Delta$ with respect to a given sequence $\left(w_{1}, \ldots, w_{m}\right)$ of elements. Suppose that

$$
\begin{aligned}
\Delta\left(w_{j},\left\{w_{1}, \ldots, w_{j-1}\right\}\right) & \sim f_{j}(t)(j \in[m]) ; \\
\Delta\left(\emptyset,\left\{w_{1}, \ldots, w_{m}\right\}\right) & \sim g(t) .
\end{aligned}
$$

Then $\Delta \sim g(t)+\sum_{j=1}^{m} f_{j}(t) t$.

Proof. We claim that $\Delta\left(\emptyset,\left\{w_{1}, \ldots, w_{i}\right\}\right) \sim g(t)+\sum_{j=i+1}^{m} f_{j}(t) t$ for $0 \leq i \leq m$; for $i=0$, we obtain the lemma. The claim is obvious for $i=m$. For $i<m$, we may assume by induction that $\Delta\left(\emptyset,\left\{w_{1}, \ldots, w_{i+1}\right\}\right) \sim g(t)+\sum_{j=i+2}^{m} f_{j}(t) t$. Since $\Delta\left(w_{i+1},\left\{w_{1}, \ldots, w_{i}\right\}\right) \sim f_{i+1}(t)$, the claim follows by Lemma 3.1.

\section{Further properties of semi-nonevasive and semi- collapsible complexes}

We examine to what extent semi-nonevasiveness and semi-collapsibility are preserved under join, barycentric subdivision, direct product, and Alexander duality. The results are either generalizations of results due to Welker [38] or generalizations of weaker results. Open problems are listed at the end of the section.

Theorem 4.1 (Welker [38]) If at least one of $\Delta$ and $\Gamma$ is collapsible (nonevasive), then the join $\Delta * \Gamma$ is collapsible (nonevasive). If $\Delta * \Gamma$ is nonevasive, then at least one of $\Delta$ and $\Gamma$ is nonevasive. 
Theorem 4.2 If $\Delta$ and $\Gamma$ are both semi-collapsible (semi-nonevasive) over $\mathbb{F}$, then the join $\Delta * \Gamma$ is semi-collapsible (semi-nonevasive) over $\mathbb{F}$. If $\Delta * \Gamma$ is semi-nonevasive over $\mathbb{F}$ and evasive, then each of $\Delta$ and $\Gamma$ is semi-nonevasive over $\mathbb{F}$ and evasive.

Proof. First, consider semi-collapsibility. If $\Delta$ satisfies $(i)$ in Definition 2.4 , then $\Delta * \Gamma$ is either $\emptyset, \Gamma$, or a cone over $\Gamma$. Each of these complexes is semi-collapsible by assumption. Suppose $\Delta$ satisfies (ii) in Definition 2.4 with shedding face $\sigma$. By assumption, fdel $\Delta(\sigma)$ and $\operatorname{link}_{\Delta}(\sigma)$ are both semi-collapsible, which implies by induction that fdel ${ }_{\Delta * \Gamma}(\sigma)$ and $\operatorname{link}_{\Delta * \Gamma}(\sigma)$ are semi-collapsible. For any complex $\Sigma$, let $\tilde{\beta}_{\Sigma}(t)=\sum_{i \geq-1} \operatorname{dim} \tilde{H}_{i}(\Sigma, \mathbb{F}) t^{i}$. By well-known properties of the join operator (see Björner [3]), we have that

$$
\begin{aligned}
\tilde{\beta}_{\Delta * \Gamma}(t) / t & =\tilde{\beta}_{\Delta}(t) \tilde{\beta}_{\Gamma}(t)=\left(\tilde{\beta}_{\Delta(\emptyset, \sigma)}(t)+t^{|\sigma|} \tilde{\beta}_{\Delta(\sigma, \emptyset)}(t)\right) \tilde{\beta}_{\Gamma}(t) \\
& =\left(\tilde{\beta}_{\Delta(\emptyset, \sigma) * \Gamma}(t)+t^{|\sigma|} \tilde{\beta}_{\Delta(\sigma, \emptyset) * \Gamma}(t)\right) / t,
\end{aligned}
$$

where the second identity follows from the fact that (3) holds for $\Delta$ and $\sigma$. Thus (3) holds for $\Delta * \Gamma$ and $\sigma$, and we are done with the first statement. Join preserving seminonevasiveness is proved in exactly the same manner.

For the second statement, suppose that $\Delta * \Gamma$ is semi-nonevasive and evasive. If $\Delta * \Gamma=\{\emptyset\}$, then we are done. Otherwise, let $x$ be the first shedding vertex; we may assume that $\{x\} \in \Delta$. Since $\Delta * \Gamma$ is evasive, either the link or the deletion (or both) with respect to $x$ is evasive. By induction, $\operatorname{del}_{\Delta}(x) * \Gamma\left(\operatorname{link}_{\Delta}(x) * \Gamma\right)$ being semi-nonevasive and evasive implies that the same holds for both $\operatorname{del}_{\Delta}(x)\left(\operatorname{link}_{\Delta}(x)\right)$ and $\Gamma$. Also, $\operatorname{del}_{\Delta}(x) * \Gamma$ $\left(\operatorname{link}_{\Delta}(x) * \Gamma\right)$ being nonevasive implies that $\operatorname{del}_{\Delta}(x)\left(\operatorname{link}_{\Delta}(x)\right)$ must be nonevasive by Theorem 4.1; $\Gamma$ is evasive by assumption. As a consequence, $\operatorname{del}_{\Delta}(x)$ and $\operatorname{link}_{\Delta}(x)$ are both semi-nonevasive. Since $\Delta * \Gamma$ and $x$ satisfy (2), we obtain that

$$
\begin{aligned}
t \tilde{\beta}_{\Delta}(t) \tilde{\beta}_{\Gamma}(t) & =\tilde{\beta}_{\Delta * \Gamma}(t)=\tilde{\beta}_{\Delta(\emptyset, x) * \Gamma}(t)+t \tilde{\beta}_{\Delta(x, \emptyset) * \Gamma}(t) \\
& =t\left(\tilde{\beta}_{\Delta(\emptyset, x)}(t)+t \tilde{\beta}_{\Delta(x, \emptyset)}(t)\right) \tilde{\beta}_{\Gamma}(t) .
\end{aligned}
$$

$\Gamma$ being semi-nonevasive and evasive implies that $\tilde{\beta}_{\Gamma}(t)$ is nonzero and hence cancels out in this equation. As a consequence, $\tilde{\beta}_{\Delta}(t)=\tilde{\beta}_{\Delta(\emptyset, x)}(t)+t \tilde{\beta}_{\Delta(x, \emptyset)}(t)$, which means exactly that (2) holds for $\Delta$ and $x$. We are thus done by induction.

Using exactly the same technique as in the proof of Theorem 4.2, one obtains the following more general result.

Theorem 4.3 With notation as in Section 3, if $\Delta \sim f(t)$ and $\Gamma \sim g(t)$, then $\Delta * \Gamma \sim$ $\operatorname{tg}(t) f(t)$. The analogous property holds for set-decision trees (i.e., acyclic matchings).

Theorem 4.4 (Welker [38]) If $\Delta$ is a collapsible simplicial complex, then the barycentric subdivision $\operatorname{sd}(\Delta)$ of $\Delta$ is nonevasive.

Theorem 4.5 If $\Delta$ is semi-collapsible over $\mathbb{F}$, then the barycentric subdivision $\operatorname{sd}(\Delta)$ of $\Delta$ is semi-nonevasive over $\mathbb{F}$. 
Remark. Theorems 4.4 and 4.5 are closely related to a theorem of Provan and Billera [30, Cor. 3.3.2] stating that $\operatorname{sd}(\Delta)$ is vertex-decomposable whenever $\Delta$ is shellable.

Proof. Throughout this proof, we will freely use the fact that homology is preserved under barycentric subdivision. Write $\Sigma=\operatorname{sd}(\Delta)$. If $\Delta$ satisfies $(i)$ in Definition 2.4, then $\Sigma$ satisfies $(i)$ in Definition 2.3. Suppose that $\Delta$ satisfies (ii) in Definition 2.4 with $\sigma$ as the shedding face. Note that

$$
\operatorname{link}_{\Sigma}(\sigma) \cong \operatorname{sd}\left(2^{\sigma} \backslash\{\sigma\}\right) * \operatorname{sd}\left(\operatorname{link}_{\Delta}(\sigma)\right)
$$

where $2^{\sigma}$ is the full simplex on the set $\sigma$. Namely, each chain in $\operatorname{link}_{\Sigma}(\sigma)$ consists of nonempty faces that are either proper subsets of $\sigma$ (i.e., contained in $2^{\sigma} \backslash\{\sigma, \emptyset\}$ ) or proper supersets of $\sigma$ (i.e., of the form $\sigma \cup \tau$ for some $\tau \in \operatorname{link}_{\Delta}(\sigma) \backslash\{\emptyset\}$ ). Since $2^{\sigma} \backslash\{\sigma\}$ and $\operatorname{link}_{\Delta}(\sigma)$ are both semi-collapsible, the corresponding barycentric subdivisions are seminonevasive by induction on the size of $\Delta$. By Theorem 4.2, this implies that $\operatorname{link}_{\Sigma}(\sigma)$ is semi-nonevasive. By properties of join, we have that

$$
\tilde{H}_{i}\left(\operatorname{link}_{\Sigma}(\sigma)\right) \cong \bigoplus_{a+b=i-1} \tilde{H}_{a}\left(2^{\sigma} \backslash\{\sigma\}\right) \otimes \tilde{H}_{b}\left(\operatorname{link}_{\Delta}(\sigma)\right) \cong \tilde{H}_{i+1-|\sigma|}\left(\operatorname{link}_{\Delta}(\sigma)\right)
$$

For the deletion $\operatorname{del}_{\Sigma}(\sigma)$, let $\tau_{1}, \ldots, \tau_{r}$ be the faces in $\Delta$ that properly contain $\sigma$, arranged in increasing order $\left(\left|\tau_{i}\right|<\left|\tau_{j}\right| \Rightarrow i<j\right)$. Consider the first-hit decomposition of $\operatorname{del}_{\Sigma}(\sigma)$ with respect to $\left(\tau_{1}, \ldots, \tau_{r}\right)$; see Definition 3.2.

We have that

$$
\Sigma\left(\tau_{i},\left\{\sigma, \tau_{1}, \ldots, \tau_{i-1}\right\}\right) \cong \operatorname{sd}\left(\operatorname{fdel}_{2^{\tau_{i}}}(\sigma)\right) * \operatorname{sd}\left(\operatorname{link}_{\Delta}\left(\tau_{i}\right)\right) .
$$

Namely, all faces $\rho$ such that $\sigma \subset \rho \subset \tau_{i}$ are among the faces $\tau_{1}, \ldots, \tau_{i-1}$ and hence deleted, whereas all faces $\rho$ such that $\tau_{i} \subset \rho$ are among the faces $\tau_{i+1}, \ldots, \tau_{r}$ and hence not yet deleted. It is clear that any element in $\tau_{i} \backslash \sigma$ is a cone point in $\operatorname{fdel}_{2^{\tau_{i}}}(\sigma)$, which implies by induction that the corresponding barycentric subdivision is nonevasive. By Theorem 4.1, it follows that $\Sigma\left(\tau_{i},\left\{\sigma, \tau_{1}, \ldots, \tau_{i-1}\right\}\right)$ is nonevasive.

Finally, $\Sigma\left(\emptyset,\left\{\sigma, \tau_{1}, \ldots, \tau_{r}\right\}\right)=\operatorname{sd}\left(\operatorname{fdel}_{\Delta}(\sigma)\right)$, which is semi-nonevasive by induction. By Lemma 3.3 (and Proposition 2.5), $\operatorname{del}_{\Sigma}(\sigma)$ is semi-nonevasive with the same homology as $\operatorname{fdel}_{\Delta}(\sigma)$. By assumption, (3) holds for $\Delta$ and $\sigma$, which implies by (5) that (2) holds for $\Sigma$ and $\sigma$, and we are done.

Before proceeding with direct products, we prove a lemma that may also be of some use in other situations. Let $\Delta$ and $\Gamma$ be families of sets. Say that a map $\varphi: \Gamma \rightarrow \Delta$ is order-preserving if $\gamma_{1} \subseteq \gamma_{2}$ implies that $\varphi\left(\gamma_{1}\right) \subseteq \varphi\left(\gamma_{2}\right)$. For $\sigma \in \Delta$, let $\Gamma_{\sigma}=\varphi^{-1}(\sigma)$.

Lemma 4.6 For nonempty finite families $\Delta$ and $\Gamma$ of finite sets, let $\mathcal{M}_{\Delta}$ be an acyclic matching on $\Delta$ and let $\varphi: \Gamma \rightarrow \Delta$ be an order-preserving map. For each critical set $\rho$ with respect to $\mathcal{M}_{\Delta}$, let $\mathcal{M}_{\rho}$ be an acyclic matching on $\Gamma_{\rho}$. For each matched pair $\{\sigma, \tau\}$ with respect to $\mathcal{M}_{\Delta}$, let $\mathcal{M}_{\sigma, \tau}$ be an acyclic matching on $\Gamma_{\sigma} \cup \Gamma_{\tau}$. Then the union $\mathcal{M}_{\Gamma}$ of all matchings $\mathcal{M}_{\rho}$ and $\mathcal{M}_{\sigma, \tau}$ is an acyclic matching on $\Gamma$. 
Remark. When $\mathcal{M}_{\Delta}$ is empty, Lemma 4.6 reduces to the Cluster Lemma; see Jonsson [20, Sec. 2].

Proof. Consider a set-decision tree $T$ corresponding to $\mathcal{M}_{\Delta}$; use Theorem 1.4. If $\Delta=\{\emptyset\}$ or $\Delta=\{\emptyset,\{v\}\}$ with $\emptyset$ and $\{v\}$ matched, then the lemma is trivial since we consider the union of one single matching. Otherwise, suppose that $T=\left(\sigma, T_{0}, T_{1}\right)$. Let $\Gamma_{D}=$ $\bigcup_{\tau \in \text { fdel }_{\Delta}(\sigma)} \Gamma_{\tau}$ and $\Gamma_{L}=\bigcup_{\tau \in \operatorname{link}_{\Delta}(\sigma)} \Gamma_{\sigma \cup \tau}$. By induction, the union of all matchings $\mathcal{M}_{\rho}$ and $\mathcal{M}_{\sigma, \tau}$ for $\rho, \sigma, \tau \in$ fdel $_{\Delta}(\sigma)$ is an acyclic matching on $\Gamma_{D}$; the analogous property also holds for $\Gamma_{L}$. Now, there are no edges directed from $\Gamma_{D}$ to $\Gamma_{L}$ in the digraph of $\mathcal{M}_{\Gamma}$. Namely, that would imply either that some $\gamma_{0} \in \Gamma_{D}$ is matched with some $\gamma_{1} \in \Gamma_{L}$ (which is impossible) or that some $\gamma_{0} \in \Gamma_{D}$ contains some $\gamma_{1} \in \Gamma_{L}$ (which contradicts the fact that $\varphi$ is order-preserving). As a consequence, $\mathcal{M}_{\Gamma}$ is acyclic.

Theorem 4.7 (Welker [38]) If $P$ and $Q$ are posets such that $\Delta(P)$ and $\Delta(Q)$ are both collapsible (nonevasive), then $\Delta(P \times Q)$ is collapsible (nonevasive). The converse is false for collapsible complexes.

Remark. One easily adapts Welker's proof of Theorem 4.7 to a proof that $\Delta(P \times Q)$ is semi-nonevasive whenever $\Delta(P)$ is nonevasive and $\Delta(Q)$ is semi-nonevasive.

Theorem 4.8 If $P$ and $Q$ are posets such that $\Delta(P)$ and $\Delta(Q)$ are both semi-collapsible over $\mathbb{F}$, then $\Delta(P \times Q)$ is semi-collapsible over $\mathbb{F}$. The converse is false.

Proof. Our goal is to construct an optimal acyclic matching on $\Gamma=\Delta(P \times Q)$ given optimal acyclic matchings $\mathcal{M}_{P}$ and $\mathcal{M}_{Q}$ on $\Delta(P)$ and $\Delta(Q)$, respectively. For technical reasons, we leave the empty set unmatched in both matchings (hence the matchings are only almost optimal). For any complex $\Sigma$, let $\beta_{\Sigma}(t)=\sum_{i>0} \operatorname{dim} H_{i}(\Sigma, \mathbb{F}) t^{i}$ (unreduced homology). Since $\Gamma$ is homotopy equivalent to the product of $\bar{\Delta}(P)$ and $\Delta(Q)$ (see Björner $[3])$, we have that $\beta_{\Gamma}(t)=\beta_{\Delta(P)}(t) \beta_{\Delta(Q)}(t)$. In particular, we want to find an acyclic matching with one critical face of size $i+j-1$ for each pair of nonempty critical faces $\sigma \in \Delta(P)$ and $\tau \in \Delta(Q)$ of size $i$ and $j$, respectively.

Let $\Pi_{P}: \Delta(P \times Q) \rightarrow \Delta(P)$ be the projection map; $\Pi_{P}\left(\left\{\left(x_{i}, y_{i}\right): i \in I\right\}\right)=\left\{x_{i}: i \in\right.$ $I\}$. For $\sigma \in \Delta(P)$, let $\Gamma_{\sigma}=\Pi_{P}^{-1}(\sigma)$. It is clear that $\Pi_{P}$ is order-preserving. Specifically, given an acyclic matching on $\Gamma_{\sigma_{1}} \cup \Gamma_{\sigma_{2}}$ for each pair $\left\{\sigma_{1}, \sigma_{2}\right\} \in \mathcal{M}_{P}$ and an acyclic matching on $\Gamma_{\rho}$ for each critical face $\rho$ with respect to $\mathcal{M}_{P}$, Lemma 4.6 yields that the union of all these matchings is an acyclic matching on $\Gamma$.

First, let us use a construction from Welker's proof [38] of Theorem 4.7 to obtain a perfect matching on $\Gamma_{\sigma_{1}} \cup \Gamma_{\sigma_{2}}$ for each $\left\{\sigma_{1}, \sigma_{2}\right\} \in \mathcal{M}_{P} ; \sigma_{2}=\sigma_{1}+x$. Since $\sigma_{2}$ contains at least two elements, $x$ is either not maximal or not minimal in $\sigma_{2}$; by symmetry, we may assume that $x$ is not maximal. Let $x^{\prime}$ be the smallest element in $\sigma_{2}$ that is larger than $x$. For a given element $\gamma$ in $\Gamma_{\sigma_{1}} \cup \Gamma_{\sigma_{2}}$ let $b_{\gamma}$ be minimal such that $\left(x^{\prime}, b_{\gamma}\right) \in \Gamma$. We obtain a perfect matching by matching $\gamma-\left(x, b_{\gamma}\right)$ with $\gamma+\left(x, b_{\gamma}\right)$. Namely, adding or removing $\left(x, b_{\gamma}\right)$ does not affect $b_{\gamma}$, and adding $\left(x, b_{\gamma}\right)$ leads to a new chain due to the minimality of $b_{\gamma}$. The matching is acyclic, as it corresponds to an element-decision tree in which we first 
query all elements $(a, b)$ such that $a \neq x$ and then query all remaining elements except $\left(x, b_{\gamma}\right)$ (which only depends on elements queried in the first round).

Next, we want to find a matching on $\Gamma_{\rho}$ for each critical face $\rho$ in $\Delta(P)$. Consider the order-preserving projection map $\Pi_{Q}: \Gamma_{\rho} \rightarrow \Delta(Q)$ and let $\Gamma_{\rho, \tau}=\Pi_{Q}^{-1}(\tau)$. By Lemma 4.6, given acyclic matchings on $\Gamma_{\rho, \tau_{1}} \cup \Gamma_{\rho, \tau_{2}}$ for $\left\{\tau_{1}, \tau_{2}\right\} \in \mathcal{M}_{Q}$ and acyclic matchings on $\Gamma_{\rho, \tau}$ for $\tau$ critical, the union of all matchings is an acyclic matching on $\Gamma_{\rho}$. We easily obtain a perfect acyclic matching on $\Gamma_{\rho, \tau_{1}} \cup \Gamma_{\rho, \tau_{2}}$ in exactly the same manner as we obtained the matching on $\Gamma_{\sigma_{1}} \cup \Gamma_{\sigma_{2}}$ above. What remains is the family $\Gamma_{\rho, \tau}$ for each pair of nonempty critical faces $\rho \in \Delta(P)$ and $\tau \in \Delta(Q)$. Write $\rho=x_{1} x_{2} \ldots x_{k}$ and $\tau=y_{1} y_{2} \ldots y_{r} ; x_{i}<x_{i+1}$ and $y_{j}<y_{j+1}$. It is clear that every face in $\Gamma_{\rho, \tau}$ contains $\left(x_{1}, y_{1}\right)$. We use induction on $k=|\rho|$ to show that there is an element-decision tree on $\Gamma_{\rho, \tau}$ with exactly one critical face of size $|\rho|+|\tau|-1$; this will yield the theorem.

For $|\rho|=1, \Gamma_{\rho, \tau}$ consists of one single face of size $|\tau|=|\rho|+|\tau|-1$. For $|\rho|>1$, note that the deletion $\Gamma_{\rho, \tau}\left(\emptyset,\left(x_{1}, y_{1}\right)\right)$ is empty; $\left(x_{1}, y_{1}\right)$ is present in every face in $\Gamma_{\rho, \tau}$. Write $\Lambda=\Gamma_{\rho, \tau}\left(\left(x_{1}, y_{1}\right), \emptyset\right)$ and proceed with the first-hit decomposition of $\Lambda$ with respect to $\left(\left(x_{2}, y_{1}\right),\left(x_{2}, y_{2}\right), \ldots,\left(x_{2}, y_{k}\right)\right)$; see Definition 3.2. We have that

$$
\Lambda\left(\left(x_{2}, y_{1}\right), \emptyset\right)=\Gamma_{\rho-x_{1}, \tau}\left(\left(x_{2}, y_{1}\right), \emptyset\right) .
$$

By induction, $\Gamma_{\rho-x_{1}, \tau}\left(\left(x_{2}, y_{1}\right), \emptyset\right)$ admits an element-decision tree with one critical face of size $|\rho|-1+|\tau|-2$. Adding $\left(x_{1}, y_{1}\right)$ and $\left(x_{2}, y_{1}\right)$ yields a face of the desired size $|\rho|+|\tau|-1$. In $\Lambda_{i}=\Lambda\left(\left(x_{2}, y_{i}\right),\left\{\left(x_{2}, y_{j}\right): j<i\right\}\right),\left(x_{1}, y_{i}\right)$ is a cone point. Namely, we may add the element without destroying the chain structure, and we may delete it, because both $x_{1}$ and $y_{i}$ are already contained in $\left(x_{1}, y_{1}\right)$ and $\left(x_{2}, y_{i}\right)$, respectively. Thus $\Lambda_{i}$ is nonevasive, and we are done by Lemma 3.3.

The final statement is an immediate consequence of Theorem 4.7.

Proposition 4.9 (Welker [38]) A simplicial complex $\Delta$ on a set $X$ is nonevasive if and only if the Alexander dual $\Delta_{X}^{*}$ is nonevasive. However, the Alexander dual of a collapsible complex is not necessarily collapsible.

Proposition 4.10 A simplicial complex $\Delta$ on a set $X$ is semi-nonevasive over $\mathbb{F}$ if and only if the Alexander dual $\Delta_{X}^{*}$ is semi-nonevasive over $\mathbb{F}$. However, the Alexander dual of a semi-collapsible complex is not necessarily semi-collapsible.

Proof. Use induction on the size of $X ; \operatorname{del}_{\Delta_{X}^{*}}(x)=\left(\operatorname{link}_{\Delta}(x)\right)_{X-x}^{*}$ and $\operatorname{link}_{\Delta_{X}^{*}}(x)=$ $\left(\operatorname{del}_{\Delta}(x)\right)_{X-x}^{*}$. By $(1),(2)$ holds for $\Delta_{X}^{*}$ if and only if it holds for $\Delta$. In the base case, we have the Alexander dual of $\emptyset,\{\emptyset\}$, or $\{\emptyset,\{v\}\}$; all three duals are easily seen to be seminonevasive over any field. For the final statement, a contractible complex is collapsible if and only if the complex is semi-collapsible. This implies by Proposition 4.9 that the Alexander dual of a semi-collapsible complex is not necessarily semi-collapsible.

Finally, we present a few important open problems; some of them are due to Welker [38].

- Is at least one of $\Delta$ and $\Gamma$ collapsible whenever $\Delta * \Gamma$ is collapsible? Is each of $\Delta$ and $\Gamma$ semi-collapsible whenever $\Delta * \Gamma$ is semi-collapsible but not collapsible? 
- Is $\Delta$ collapsible (semi-collapsible) whenever the barycentric subdivision of $\Delta$ is nonevasive (semi-nonevasive)? Is there a complex $\Delta$ such that the barycentric subdivision of $\Delta$ is collapsible or semi-collapsible but not semi-nonevasive?

- Are $\Delta(P)$ and $\Delta(Q)$ both nonevasive whenever $\Delta(P \times Q)$ is nonevasive? Is $\Delta(P \times Q)$ semi-nonevasive whenever both $\Delta(P)$ and $\Delta(Q)$ are semi-nonevasive and evasive?

\section{$5 \quad$ Examples}

This section contains a list of well-known complexes. For each complex, we show how to use decision trees to determine the homotopy type and homology; earlier proofs can be found in the literature $[30,5,4,2,37,33]$. As a byproduct, we obtain that the complexes under consideration are semi-nonevasive over $\mathbb{Z}$. The matching complex in Proposition 5.5 constitutes an exception, as we can only determine partial information about its topology.

In most cases, we consider complexes of graphs or digraphs. Fixing the underlying vertex set $V$, we may identify a graph or a digraph with its edge set. In particular, if a family of graphs or digraphs on the vertex set $V$ is closed under deletion of edges, then we may view the family as a simplicial complex. Some of our complexes are invariant under the natural action of the symmetric group on $V$. We refer to such complexes as monotone (di-)graph properties.

Our initial examples in Section 5.1 are quite simple; the purpose is to present some approaches for defining decision trees. Section 5.2 is devoted to more complicated complexes defined in terms of cycles in digraphs. In Sections 5.3 and 5.4, we proceed with complexes of graphs containing small connected components such as isolated edges and vertices. Theorem 5.7 in Section 5.4 - to our knowledge a new result - unifies the properties of being disconnected and containing isolated vertices. The exhibition is concluded in Section 5.5, where we examine not 2-connected graphs.

We concentrate on element-decision trees and do not consider more general set-decision trees. An interesting question is whether set-decision trees may provide a fruitful tool for proving semi-collapsibility analogously to the way we use element-decision trees to prove semi-nonevasiveness. An important difference between the two notions is that there are other powerful methods available (e.g., explicit acyclic matchings) to obtain semi-collapsibility, whereas it seems that any proof of semi-nonevasiveness must go, in one way or another, via element-decision trees.

\subsection{Warming up}

Our first example is a proof of the well-known fact that (Alexander duals of) matroid complexes have a nice homotopy type. For more information about matroids, see Oxley [29] and Welsh [39].

Proposition 5.1 Let $k \geq 0$, let $M$ be a matroid on a nonempty set $X$, and let $\Sigma_{M, k}$ be the complex of all sets of rank at most $k$. Then $\Sigma_{M, k} \sim c t^{k-1}$ for some $c \geq 0$ (notation as in Section 3). Hence $\Sigma_{M, k}$ is homotopy equivalent to a wedge of $(k-1)$-spheres. 
Proof. The statement is easy to check if $X$ consists of one single element. Suppose that $|X| \geq 2$ and let $x$ be any element in $X$. If the rank of $x$ is 0 or the rank of $M$ is at most $k$, then $x$ is a cone point, which implies that $\Sigma_{M, k} \sim 0$. Otherwise, $\Sigma_{M, k}(\emptyset, x)=\Sigma_{M-x, k}$ and $\Sigma_{M, k}(x, \emptyset)=\Sigma_{M / x, k-1}$, where $M / x$ denotes contraction with respect to $x ; \operatorname{rank}_{M / x}(S)=$ $\operatorname{rank}_{M}(S+x)-1$. Note that $\Sigma_{M / x, k-1}$ is void if $k=0$. By induction, $\Sigma_{M, k}(\emptyset, x) \sim c_{\emptyset} t^{k-1}$ and $\Sigma_{M, k}(x, \emptyset) \sim c_{x} t^{k-2}$ for some $c_{\emptyset}, c_{x} \geq 0$; thus we are done by Lemma 3.1.

Remark. The Alexander dual of $\Sigma_{M, k}$ is vertex-decomposable; see Provan and Billera [30]. Proposition 5.1 is hence a consequence of Propositions 4.10 and 2.12.

In our second example, we consider simple graphs, i.e., 1-dimensional complexes.

Proposition 5.2 Let $G=(V, E)$ be a simple connected graph with e edges and $n$ vertices. Then $G \sim(e-n+1) t$.

Proof. $G$ is clearly nonevasive if $G$ has one vertex. Suppose that $G$ has at least two vertices. Let $v$ be a vertex such that the induced subgraph $G^{\prime}=G(\emptyset, v)=\operatorname{del}_{G}(v)$ obtained by removing $v$ is connected; let $v$ be a leaf in a spanning tree. By induction, we obtain that $G(\emptyset, v) \sim\left(e-\left|N_{v}\right|-(n-1)+1\right) t$. Moreover, $\operatorname{link}_{G}(v)=G(v, \emptyset)$ consists of the empty set and the vertices in $N_{v}=\{w: v w \in G\}$; clearly, $\operatorname{link}_{G}(v) \sim\left(\left|N_{v}\right|-1\right)$. By Lemma 3.1, $G \sim\left(\left|N_{v}\right|-1\right) t+\left(e-\left|N_{v}\right|-n+2\right) t=(e-n+1) t$ as desired.

\section{$5.2 \quad$ Directed cycles}

A digraph $D$ is strongly connected if every pair of vertices in $D$ is contained in a directed cycle. Recall that $D$ is acyclic if $D$ contains no directed cycles. Let $\Delta_{n}^{N S C}$ be the complex of not strongly connected digraphs on the vertex set $[n]$, and let $\Delta_{n}^{A C Y}$ be the complex of acyclic digraphs on $[n]$. The homotopy type of $\Delta_{n}^{N S C}$ and $\Delta_{n}^{A C Y}$ was first determined by Björner and Welker [5].

Theorem 5.3 For $n \geq 2, \Delta_{n}^{N S C} \sim(n-1) ! \cdot t^{2 n-4}$. Hence $\Delta_{n}^{N S C}$ is homotopy equivalent to a wedge of $(n-1)$ ! spheres of dimension $2 n-4$.

Proof. We use induction on $n$. The case $n=2$ is easy to check; assume that $n>2$. Let $A=\bigcup_{i \in[n-1]}\{(i, n),(n, i)\}$, and consider the complex $\Sigma_{Y}=\Delta_{n}^{N S C}(Y, A \backslash Y)$ for each $Y \subseteq A$. If $Y$ does not contain any edge $(i, n)$, then $\Sigma_{Y}$ is nonevasive; any element in $\Sigma_{Y}$ is a cone point, as the underlying digraph cannot be strongly connected. The similar property holds if $Y$ does not contain any edge $(n, i)$. Moreover, if $(i, n),(n, j) \in Y$ for some distinct $i, j$, then the edge $(i, j)$ is a cone point in $\Sigma_{Y}$; we already have a directed path from $i$ to $j$ via $n$.

The remaining sets $Y$ are of the form $\{(i, n),(n, i)\}$. We claim that $\Sigma_{Y}=\Delta_{n-1}^{N S C}$ for each such $Y$. Namely, a digraph $D$ containing $Y$ but not $A \backslash Y$ is clearly strongly connected if and only if the digraph obtained from $D$ by removing the vertex $n$ along with 
the edges $(n, i)$ and $(i, n)$ is strongly connected. By induction, $\Delta_{n-1}^{N S C} \sim(n-2) ! \cdot t^{2 n-6}$, which by Lemma 3.1 implies that

$$
\Delta_{n}^{N S C} \sim \sum_{i=1}^{n-1}(n-2) ! \cdot t^{2 n-6} \cdot t^{2}=(n-1) ! \cdot t^{2 n-4},
$$

and we are done.

Theorem 5.4 For $n \geq 1, \Delta_{n}^{A C Y} \sim t^{n-2}$. Hence $\Delta_{n}^{A C Y}$ is homotopy equivalent to the $(n-2)$-sphere.

Proof. We use induction on $n$. For $n=1$, we have that $\Delta_{1}^{A C Y}=\{\emptyset\}$; assume that $n>1$. Consider the first-hit decomposition of $\Delta_{n}^{A C Y}$ with respect to $((1, n),(2, n), \ldots,(n-$ $1, n))$; see Definition 3.2. For $r \in[n-1]$, let $A_{r}=\{(i, n): i \in[r]\}$. Let $\Sigma_{r}=$ $\Delta_{n}^{A C Y}\left(\{(r, n)\}, A_{r-1}\right)$ and $\Sigma_{n}=\Delta_{n}^{A C Y}\left(\emptyset, A_{n-1}\right)$. We want to show that $\Sigma_{r}$ is nonevasive for $r \neq n-1$ and that $\Sigma_{n-1} \sim t^{n-3}$. By Lemma 3.3, it then follows that $\Delta_{n}^{A C Y} \sim t^{n-3} \cdot t=t^{n-2}$.

Clearly, $(n, i)$ is a cone point in $\Sigma_{n}$ for any $i$; if no edges are directed to $n$, then $n$ cannot be contained in a cycle. For $r \leq n-1$, let $B=\{(n, i): i \in[n-1]\}$. For each $Z \subseteq B$, consider the complex $\Sigma_{r, Z}=\Sigma_{r}(Z, B \backslash Z)$. If $(n, r) \in Z$, then $\Sigma_{r, Z}$ is void; $((n, r),(r, n))$ is a cycle. If $(n, i) \in Z$ for some $i \neq r$, then $(r, i)$ is a cone point in $\Sigma_{r, Z}$; we already have a directed path from $r$ to $i$ via $n$.

What remains is to consider $Z=\emptyset$. If $r \neq n-1$, then $(r+1, n)$ is a cone point in $\Sigma_{r, \emptyset} ; n$ cannot be contained in a cycle since there are no edges directed from $n$. As a consequence, $\Sigma_{r} \sim 0$ if $r \neq n-1$ by Lemma 3.1; $\Sigma_{r, Z} \sim 0$ for all $Z$. For $r=n-1$, we have that $\Sigma_{n-1, \emptyset}=\Delta_{n-1}^{A C Y}$. Namely, a digraph $D$ containing $(n-1, n)$ but no other edges incident to $n$ is clearly acyclic if and only if the digraph obtained from $D$ by removing the vertex $n$ along with the edge $(n-1, n)$ is acyclic. By induction, $\Delta_{n-1}^{A C Y} \sim t^{n-3}$, which by Lemma 3.1 implies that $\Sigma_{n-1} \sim t^{n-3}$, and we are done.

\subsection{Matching complexes}

The matching complex $M_{n}$ is the family of all graphs $G$ on the vertex set $[n]$ with pairwise disjoint edges. The rational homology of $M_{n}$ was completely determined by Bouc [9]. Athanasiadis [1] proved that the $\nu_{n}$-skeleton of $M_{n}$ is vertex-decomposable, where $\nu_{n}=$ $\left\lceil\frac{n-4}{3}\right\rceil$. By Proposition 2.12, this implies that there is an element-decision tree on $M_{n}$ such that the dimension of each evasive face is at least $\nu_{n}$. Indeed, such a tree is easy to define:

Proposition 5.5 Let $n \geq 1$. Then $M_{n} \sim \sum_{j \geq 0} c_{j} t^{j}$, where $c_{j}=0$ unless

$$
\left\lceil\frac{n-4}{3}\right\rceil \leq j \leq\left\lfloor\frac{n-3}{2}\right\rfloor \text {. }
$$

Hence $M_{n}$ is homotopy equivalent to a $C W$ complex in which all cells (except one 0-cell) have dimensions in the interval (6). 
Proof. For $n \leq 3, M_{n}$ consists of the empty set and all singleton sets. Assume that $n \geq 4$ and consider the first-hit decomposition of $M_{n}$ with respect to the sequence $(13,14, \ldots, 1 n, 23,24, \ldots, 2 n)$. We immediately note that $M_{n}(\emptyset, E) \sim 0$, where $E=$ $\{1 i, 2 i: i \in\{3, \ldots, n\}\}$. Namely, 12 is a cone point, as $E$ contains all edges intersecting 12 .

Now, consider $\Sigma_{r}=M_{n}\left(\left\{e_{r}\right\},\left\{e_{1}, \ldots, e_{r-1}\right\}\right)$, where $e_{r}=a b$ is the $r$ th element in the sequence under consideration; $a=1$ if $r \leq n-2$ and $a=2$ otherwise. We have that $\Sigma_{r}$ is isomorphic to $M_{n-a-1}$. Namely, if $a=1$, then all edges contained in $[n] \backslash\{1\}$ remain to be checked. If $a=2$, then all edges contained in $[n] \backslash\{1,2\}$ remain to be checked and all edges containing 1 have been deleted (except 12, but this edge intersects $2 k$ ).

By induction, $M_{n-a-1} \sim \sum_{i \geq 0} c_{i}^{\prime} t^{i}$, where $c_{i}^{\prime}=0$ unless $\left\lceil\frac{n-a-5}{3}\right\rceil \leq i \leq\left\lfloor\frac{n-a-4}{2}\right\rfloor$. Since

$$
\begin{aligned}
& \left\lceil\frac{n-a-5}{3}\right\rceil \geq\left\lceil\frac{n-7}{3}\right\rceil=\left\lceil\frac{n-4}{3}\right\rfloor-1 \\
& \left.\mid \frac{n-a-4}{2}\right\rfloor \leq\left\lfloor\frac{n-5}{2}\right\rfloor=\left\lfloor\frac{n-3}{2}\right\rfloor-1
\end{aligned}
$$

we are done by Lemma 3.3.

The second half of Proposition 5.5 was first proved by Björner, Lovász, Vrećica, and Živaljević [4]. Using results of Bouc [9], Shareshian and Wachs [34] proved that the homology group $\tilde{H}_{\nu_{n}}\left(M_{n} ; \mathbb{Z}\right)$ is nonzero for all $n \geq 3$. In particular, there is no decision tree such that the dimension of each evasive face is strictly greater than $\nu_{n}$.

Nevertheless, it is not hard to prove that the element-decision tree presented above is not optimal in general. Yet, we have at least achieved the following:

Theorem 5.6 $M_{n}$ is semi-nonevasive over $\mathbb{Z}$ for $n \leq 6$ and $n=8$. Moreover, $M_{7}$ is semi-nonevasive over $\mathbb{Z}_{3}$, but not over $\mathbb{Z}$.

Proof. The first part of the theorem is straightforward from the bounds in (6); the two bounds coincide for $3 \leq n \leq 6$ and for $n=8$.

For the second part of the theorem, the nonvanishing homology groups of $M_{7}$ are $\tilde{H}_{1}\left(M_{7} ; \mathbb{Z}\right) \cong \mathbb{Z}_{3}$ and $\tilde{H}_{2}\left(M_{7} ; \mathbb{Z}\right) \cong \mathbb{Z}^{20} ;$ see Bouc $[9]$. This means that $\tilde{H}_{1}\left(M_{7} ; \mathbb{Z}_{3}\right) \cong \mathbb{Z}_{3}$ and $\tilde{H}_{2}\left(M_{7} ; \mathbb{Z}_{3}\right) \cong \mathbb{Z}_{3}^{21}$. We want to find an element-decision tree with $1+21$ evasive faces. For this, consider the first-hit decomposition with respect to the sequence

$$
(13,14,35,46,56,12,57,67,23,24,37,47,16,25,17,27,34,36,45,15) \text {; }
$$

all edges but 26 are contained in the sequence. Let $b_{i}$ be the $i$ th edge in the sequence and let $B_{k}=\left\{b_{i}: i \leq k\right\}$. It is easy to check that $M_{7}\left(b_{i}, B_{i-1}\right) \sim c_{i}$ t for $1 \leq i \leq 6$, where $c_{1}=c_{2}=6, c_{3}=4, c_{4}=c_{5}=2$, and $c_{6}=1$; all these links are connected graphs, so optimal element-decision trees exist by Proposition 5.2. Moreover, $M_{7}\left(b_{18}, B_{17}\right) \sim 1$, whereas $M_{7}\left(\emptyset, B_{20}\right) \sim 0$ and $M_{7}\left(b_{i}, B_{i-1}\right) \sim 0$ for $i \in\{7, \ldots, 17\} \cup\{19,20\}$. Applying Lemma 3.3, we obtain that $M_{7} \sim t+21 t^{2}$ as desired. 
For $p \neq 3$, nobody has been able to detect $p$-torsion in the homology of any matching complex $M_{n}$. A related question is whether $M_{n}$ is semi-nonevasive over $\mathbb{Z}_{3}$ when $n \geq 9$; an affirmative answer for general $n$ would be a striking result. An obvious consequence of such a result would be that

$$
\tilde{H}_{d}\left(M_{n} ; \mathbb{Z}_{3}\right) \cong \tilde{H}_{d}\left(\operatorname{del}_{M_{n}}(e) ; \mathbb{Z}_{3}\right) \oplus \tilde{H}_{d-1}\left(\operatorname{link}_{M_{n}}(e) ; \mathbb{Z}_{3}\right)
$$

for all $d, n$ and any edge $e$. Since $\operatorname{link}_{M_{n}}(e) \cong M_{n-2}$, we would thus obtain an embedding of the $\mathbb{Z}_{3}$-homology of $M_{n-2}$ into the $\mathbb{Z}_{3}$-homology of $M_{n}$. One may also ask whether

$$
\tilde{H}_{d}\left(M_{n} ; \mathbb{Z}\right) \cong \tilde{H}_{d}\left(\operatorname{del}_{M_{n}}(e) ; \mathbb{Z}\right) \oplus \tilde{H}_{d-1}\left(\operatorname{link}_{M_{n}}(e) ; \mathbb{Z}\right)
$$

for all $d, n$ and any edge $e$. Using computer $[13,26]$, we have verified this to be true for $n \leq 11$.

\subsection{Graphs with small components}

In this section, we consider graphs with connected components of restricted size. One special case is the complex of graphs on the vertex set $[n]$ with at least $s$ isolated vertices. Another special case is the complex of disconnected graphs on $[n]$.

For weakly increasing sequences $\lambda=\left(\lambda_{1}, \ldots, \lambda_{l}\right)$ and $\mu=\left(\mu_{1}, \ldots, \mu_{m}\right)$ (i.e., $\lambda_{1} \leq$ $\ldots \leq \lambda_{l}$ and $\left.\mu_{1} \leq \ldots \leq \mu_{m}\right)$, say that $\lambda \leq \mu$ if $l \geq m$ and $\lambda_{i} \leq \mu_{i}$ for $1 \leq i \leq m$. Let $\omega=\left(\omega_{1}, \ldots, \omega_{n}\right)$ be a sequence of positive integers; for $S \subseteq[n]$, let

$$
\omega_{S}=\sum_{s \in S} \omega_{s}
$$

For a graph $G$ on the vertex set $[n]$ consisting of $k$ connected components, let the corresponding vertex sets $V_{1}, \ldots, V_{k}$ be ordered such that $\omega_{V_{1}} \leq \ldots \leq \omega_{V_{k}}$. Define

$$
\omega(G)=\left(\omega_{V_{1}}, \ldots, \omega_{V_{k}}\right)
$$

Let $\mu=\left(\mu_{1}, \ldots, \mu_{m}\right)$ be a (not necessarily nonempty) weakly increasing sequence of positive integers such that $\sum_{i} \mu_{i}<\sum_{j} \omega_{j}$; we say that $(\mu, \omega)$ is a permitted pair on $n$ vertices if this condition is satisfied. Let $\Lambda_{\omega}^{\mu}$ be the family of graphs $G$ on the vertex set $[n]$ satisfying $\omega(G) \leq \mu$. The family $\Lambda_{\omega}^{\mu}$ is a simplicial complex, but $\Lambda_{\omega}^{\mu}$ need not be a

graph property unless all $\omega_{i}$ are the same. Note that $\Lambda_{1^{n}}^{(n-1)}$ is the complex of disconnected graphs, where $1^{n}$ is the sequence of $n$ ones. Moreover, the complex of graphs with $s$ isolated vertices coincides with $\Lambda_{1^{n}}^{1^{s}}$. Let $L(\mu)$ be the length of the sequence $\mu ; L\left(\mu_{1}, \ldots, \mu_{m}\right)=m$.

Theorem 5.7 Let $(\mu, \omega)$ be a permitted pair. Then $\Lambda_{\omega}^{\mu} \sim c t^{n-2-L(\mu)}$ for some $c \geq 0$. Hence $\Lambda_{\omega}^{\mu}$ is homotopy equivalent to a wedge of spheres of dimension $n-2-L(\mu)$ (or void). 
Proof. Use double induction over $n$ and $\mu$. If $\mu$ is the empty sequence $\emptyset$, then $\Lambda_{\omega}^{\emptyset}$ is the full simplex on $\left(\begin{array}{l}n \\ 2\end{array}\right)$ elements and hence nonevasive unless $n=1$. If $n=1$, then $\Lambda_{\omega}^{\emptyset} \sim t^{-1}=t^{n-2-L(\mu)}$ as desired. Now, consider $n \geq 2$ and $\mu$ nonempty. Take a vertex $k$ such that $\omega_{k}$ is as small as possible; we may assume that $k=n$. If $\omega_{n}>\mu_{1}$, then $\Lambda_{\omega}^{\mu}$ is void. Otherwise, let $A=\{i n: i \in[n-1]\}$ and consider the complex $\Sigma_{Y}=\Lambda_{\omega}^{\mu}(Y, A \backslash Y)$ for each $Y \subseteq A$.

If $Y$ contains two edges $i n$ and $j n$, then $i j$ is a cone point in $\Sigma_{Y} ; i$ and $j$ are contained in the same component. Hence $\Sigma_{Y} \sim 0$. If $Y=\emptyset$, then $\Sigma_{\emptyset}=\Lambda_{\omega^{\prime}}^{\mu^{\prime}}$, where $\mu^{\prime}=\left(\mu_{2}, \ldots, \mu_{m}\right)$ and $\omega^{\prime}=\left(\omega_{1}, \ldots, \omega_{n-1}\right)$. Namely, a graph $G$ with the vertex $n$ isolated is contained in $\Lambda_{\omega}^{\mu}$ if and only if $\omega_{n} \leq \mu_{1}$ and $\omega(G(\emptyset, n)) \leq \mu^{\prime}$. Since $\mu_{1} \geq \omega_{n},\left(\mu^{\prime}, \omega^{\prime}\right)$ is a permitted pair. By induction, $\Sigma_{\emptyset}=\Lambda_{\omega^{\prime}}^{\mu^{\prime}} \sim c_{\emptyset} t^{n-3-L\left(\mu^{\prime}\right)}=c_{\emptyset} t^{n-2-L(\mu)}$ for some $c_{\emptyset} \geq 0$.

The case remaining is $\Sigma_{\{k n\}}$ for each $k \in[n-1]$. Modify $\omega$ by removing $\omega_{n}$ and by replacing $\omega_{k}$ with $\omega_{k}+\omega_{n}$. This new $\omega^{\prime}$ has the property that a graph $G$ in which $k$ is the only neighbor of $n$ is in $\Lambda_{\omega}^{\mu}$ if and only if $G(\emptyset, n) \in \Lambda_{\omega^{\prime}}^{\mu}$. Clearly, $\left(\mu, \omega^{\prime}\right)$ is a permitted pair. By induction, $\Sigma_{\{k n\}} \sim c_{k} t^{(n-1)-2-L(\mu)}$ for some $c_{k} \geq 0$. By Lemma 3.1, we obtain that

$$
\Lambda_{\omega}^{\mu} \sim c_{\emptyset} t^{n-2-L(\mu)}+\sum_{k=1}^{n-1} c_{k} t^{(n-1)-2-L(\mu)} \cdot t=c t^{n-2-L(\mu)},
$$

where $c=c_{\emptyset}+\sum_{k=1}^{n-1} c_{k}$.

Corollary 5.8 For $1 \leq s \leq n-1$, the complex $\Lambda_{1 n}^{1^{s}}$ of graphs on $n$ vertices with at least $s$ isolated vertices satisfies $\Lambda_{1^{n}}^{1^{s}} \sim\left(n\left(\begin{array}{c}n-2 \\ s-1\end{array}\right)-\left(\begin{array}{c}n-1 \\ s-1\end{array}\right)\right) t^{n-s-2}$.

Proof. By Theorem 5.7, it suffices to show that the reduced Euler characteristic $\tilde{\chi}\left(\Lambda_{1^{n}}^{1^{s}}\right)$ equals $\left(n\left(\begin{array}{c}n-2 \\ s-1\end{array}\right)-\left(\begin{array}{c}n-1 \\ s-1\end{array}\right)\right)(-1)^{n-s}$. By Quillen's Fiber Lemma (see Björner [3, Theorem 10.5]), $\Lambda_{1^{n}}^{1^{s}}$ has the same homotopy type as $\Delta\left(P_{2, n-s}^{[n]}\right)$, where $P_{a, b}^{X}$ is the poset of all subsets of the set $X$ of size between $a$ and $b$ inclusively. Namely, map a given nonempty graph to the set of vertices that are not isolated in the graph; this map clearly satisfies the conditions of Quillen's lemma. We claim that

$$
\tilde{\chi}\left(\Delta\left(P_{2, n-s}^{[n]}\right)\right)=\tilde{\chi}\left(\Delta\left(P_{1, n-s}^{[n]}\right)\right)+\sum_{i=1}^{n} \tilde{\chi}\left(\Delta\left(P_{1, n-s-1}^{[n] \backslash\{i\}}\right)\right) .
$$

Namely, $\Delta\left(P_{1, n-s}^{[n]}\right) \backslash \Delta\left(P_{2, n-s}^{[n]}\right)$ contains all chains in $P_{1, n-s}^{[n]}$ with a singleton set $\{i\}$ at the bottom for some $i \in[n]$; it is easy to see for each $i$ that we have one such chain of length $k+1$ for each chain in $P_{1, n-s-1}^{[n] \backslash\{i\}}$ of length $k$. Now, $\tilde{\chi}\left(\Delta\left(P_{1, n-s}^{[n]}\right)\right)=(-1)^{n-s-1}\left(\begin{array}{l}n-1 \\ s-1\end{array}\right)$ and $\tilde{\chi}\left(\Delta\left(P_{1, n-s-1}^{[n] \backslash\{i\}}\right)\right)=(-1)^{n-s-2}\left(\begin{array}{c}n-2 \\ s-1\end{array}\right) ; \Delta\left(P_{1, b}^{X}\right)$ is the barycentric subdivision of the $(b-1)$ skeleton of the full simplex on the set $X$. The desired result follows.

Corollary 5.9 For $n \geq 2$, the complex $\Delta_{n}^{1}=\Lambda_{1^{n}}^{(n-1)}$ of disconnected graphs on $n$ vertices satisfies $\Delta_{n}^{1} \sim(n-1) ! \cdot t^{n-3}$. 
Proof. The corollary is clearly true for $n=2$; assume that $n \geq 3$. We have that $\Delta_{n}^{1} \sim c t^{n-3}$, where $c=c_{\emptyset}+\sum_{k=1}^{n-1} c_{k}$ (notation as in the proof of Theorem 5.7). Now, $c_{\emptyset}$ equals the Euler characteristic (up to sign) of $\Lambda_{1^{n}}^{\emptyset}$, which is the full simplex and hence collapsible. Moreover, $c_{k}$ equals the Euler characteristic (up to sign) of $\Lambda_{\left(1^{n-2}, 2\right)}^{(n-1)}$. However, $\Lambda_{\left(1^{n-2}, 2\right)}^{(n-1)}$ is clearly equal to $\Lambda_{1^{n-1}}^{(n-2)}=\Delta_{n-1}^{1}$. By induction on $n, \Delta_{n-1}^{1} \sim(n-2) ! \cdot t^{n-4}$. Thus $c_{k}=(n-2) !$ and $c=\sum_{i=1}^{n-1}(n-2) !=(n-1) !$, which concludes the proof.

Remark. There are plenty of other methods for computing the homotopy type of $\Delta_{n}^{1}$; see Babson et al. [2] for references.

\subsection{Complexes of not 2-connected graphs}

As a final application, we consider complexes of not 2 -connected graphs. For $1 \leq k \leq n-1$, a graph $G$ on the vertex set $[n]$ is $k$-connected if the induced subgraph $G(\emptyset, W)$ is connected for each set $W \subset[n]$ with at most $k-1$ elements. Let $\Delta_{n}^{k}$ be the simplicial complex of not $k$-connected graphs on the vertex set $[n]$. We considered $\Delta_{n}^{1}$ in the preceding section; see Corollary 5.9

The homotopy type of $\Delta_{n}^{2}$ was discovered by Babson, Björner, Linusson, Shareshian, and Welker [2] and by Turchin [37]. Shareshian [33] gave a third proof using discrete Morse theory. Our proof is quite short, but it should be noticed that previous proofs contain much more information about the complex than just the homotopy type. For example, Shareshian [33] was able to construct an explicit basis for the homology of the quotient complex of 2-connected graphs.

Theorem 5.10 For $n \geq 3, \Delta_{n}^{2} \sim(n-2) ! \cdot t^{2 n-5}$. Hence $\Delta_{n}^{2}$ is homotopy equivalent to a wedge of $(n-2)$ ! spheres of dimension $2 n-5$.

Proof. Let $E_{n}=\{i n: i \in[n-1]\}$ and consider the complex $\Sigma_{Y}=\Delta_{n}^{2}\left(Y, E_{n} \backslash Y\right)$ for each $Y \subseteq E_{n}$. If $|Y| \leq 1$, then the degree of the vertex $n$ is at most one. In particular, any edge $i j$ such that $i, j \neq n$ is a cone point in $\Sigma_{Y}$, which implies that $\Sigma_{Y}$ is nonevasive.

From now on, assume that $|Y| \geq 2$. First, we claim that $\Sigma_{E_{n}}$ coincides with the complex $\Delta_{n-1}^{1}$ of disconnected graphs on $n-1$ vertices. Namely, since $n$ is adjacent to all other vertices in a graph $G$ containing $E_{n}, n$ is the only possible cut point; clearly, $n$ is a cut point if and only if $G(\emptyset, n)$ is disconnected. By Corollary 5.9, $\Delta_{n-1}^{1} \sim(n-2) ! \cdot t^{n-4}$. As a consequence, if we can prove that $\Sigma_{Y}$ is nonevasive whenever $Y \varsubsetneqq E_{n}$, then it follows that $\Delta_{n}^{2} \sim(n-2) ! \cdot t^{\left|E_{n}\right|} \cdot t^{n-4}=(n-2) ! \cdot t^{2 n-5}$ by Lemma 3.1.

Now, for a given set $Y \varsubsetneqq E_{n}$ such that $|Y| \geq 2$, define $\pi(Y)=\{i$ : in $\in Y\}$ and $B_{Y}=\left(\begin{array}{c}{[n-1]} \\ 2\end{array}\right) \backslash\left(\begin{array}{c}\pi(Y) \\ 2\end{array}\right)$. Consider the complex $\Sigma_{Y, Z}=\Sigma_{Y}\left(Z, B_{Y} \backslash Z\right)$ for each possible edge set $Z \subseteq B_{Y}$. Adding the edge set $Y \cup Z$ to every graph in $\Sigma_{Y, Z}$, we obtain the family of graphs containing the edge set $E=Y \cup Z$ but not any edges from $E_{n} \backslash Y$ or $B_{Y} \backslash Z$. There are three possibilities for the graph $G=([n], E)$.

- $G$ is disconnected. Since any two vertices $w_{1}, w_{2} \in \pi(Y)$ already belong to the same component in $G, w_{1} w_{2}$ is a cone point in $\Sigma_{Y, Z}$. 
- $G$ is connected, and some cycle contains the vertex $n$. Let $w_{1}, w_{2} \in \pi(Y)$ be the neighbors of $n$ in this cycle. It is clear that adding or deleting $w_{1} w_{2}$ to or from a face in $\Sigma_{Y, Z}$ does not affect the 2-connectivity of the corresponding graph; thus $w_{1} w_{2}$ is a cone point.

- $G$ is connected, and no cycle contains the vertex $n$. Let $w_{1} \in \pi(Y)$ be such that $n$ is not the only neighbor of $w_{1}$ in $G$; such a $w_{1}$ exists since $G$ is connected and fewer than $n-1$ vertices are adjacent to $n$. Let $v \neq n$ be a neighbor of $w_{1}$ in $G$. We claim that $w_{1}$ is a cut point in $G$ separating $v$ from $\{n\} \cup\left(\pi(Y) \backslash\left\{w_{1}\right\}\right)$. Namely, if there were a path from $v$ to $n$ not using $w_{1}$, then this path would form a cycle together with $w_{1}$. Since we may extend a path ending in $\pi(Y) \backslash\left\{w_{1}\right\}$ to a path ending in $n$, the claim follows. In particular, $w_{1} w_{2}$ is a cone point in $\Sigma_{Y, Z}$ for any $w_{2} \in \pi(Y) \backslash\left\{w_{1}\right\}$.

As a consequence, $\Sigma_{Y, Z}$ is always nonevasive, which by Lemma 3.1 implies that $\Sigma_{Y}$ is nonevasive; thus we are done.

Remark. In an earlier paper [20], we demonstrated that the complex $\Delta_{n}^{3}$ of not 3-connected graphs is semi-collapsible and homotopy equivalent to a wedge of $(n-3) \cdot(n-2) ! / 2$ spheres of dimension $2 n-4$. We conjecture that $\Delta_{n}^{3}$ is semi-nonevasive, i.e., $\Delta_{n}^{3} \sim$ $(n-3) \cdot(n-2) ! / 2 \cdot t^{2 n-4}$.

\section{Concluding remarks}

A potential generalization of the concept of semi-collapsibility might be as follows:

Definition 6.1 Let $\mathcal{C}$ be a family of simplicial complexes. The class of $\mathcal{C}$-collapsible simplicial complexes over the field $\mathbb{F}$ is defined recursively as follows:

(i) The void complex $\emptyset$ and any complex isomorphic to a complex in $\mathcal{C}$ are $\mathcal{C}$-collapsible over $\mathbb{F}$.

(ii) If $\Delta$ contains a nonempty face $\sigma$ such that $\operatorname{link}_{\Delta}(\sigma)$ and $\operatorname{fdel}_{\Delta}(\sigma)$ are $\mathcal{C}$-collapsible over $\mathbb{F}$ and such that

$$
\tilde{H}_{d}(\Delta ; \mathbb{F}) \cong \tilde{H}_{d}\left(\operatorname{fdel}_{\Delta}(\sigma) ; \mathbb{F}\right) \oplus \tilde{H}_{d-|\sigma|}\left(\operatorname{link}_{\Delta}(\sigma) ; \mathbb{F}\right)
$$

for each $d$, then $\Delta$ is $\mathcal{C}$-collapsible over $\mathbb{F}$.

$\mathcal{C}$-nonevasive complexes are defined analogously. Note that if $\mathcal{C}$ consists of $\{\emptyset,\{v\}\}$, then we obtain the collapsible complexes, whereas the family containing $\{\emptyset\}$ and $\{\emptyset,\{v\}\}$ yields the semi-collapsible complexes. We do not know whether the given generalization leads to anything useful.

For a given family of simplicial complexes, a natural problem is to characterize those complexes in the family that are semi-collapsible or semi-nonevasive, say over $\mathbb{Z}$. For 


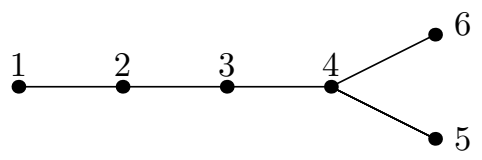

Figure 5: The graph with edge set $\{12,23,34,45,46\}$.

example, let us consider monotone graph properties. Karp's famous evasiveness conjecture states that there are no nonevasive monotone graph properties except the void complex and the full simplex. The conjecture was partially settled by Kahn, Saks, and Sturtevant [21]; see the work of Chakrabarti, Khot, and Shi [10] for some recent progress. An interesting question related to the evasiveness conjecture is whether there are collapsible monotone graph properties that are not nonevasive. More generally, one may ask whether there are semi-collapsible monotone graph properties that are not semi-nonevasive. Not surprisingly, the answer to the second question is yes:

Let $\Delta$ be the complex of all graphs on the vertex set $\{1,2,3,4,5\}$ that are contained in a copy of $\{12,34,35\}$. This complex is collapsible to the matching complex $M_{5}$ on five vertices (see Section 5.3); collapse all pairs $(\{c d, c e\},\{a b, c d, c e\})$. Since $M_{5}$ is semicollapsible by Theorem 5.6, the same is true for $\Delta$. However, $\Delta$ is not semi-nonevasive. Namely, the three 1-cells $\{34,35\},\{34,45\},\{35,45\}$ form a cycle in $\operatorname{link}_{\Delta}(12)$, which implies that $\operatorname{link}_{\Delta}(12)$ has nonvanishing homology in its top dimension; by symmetry, the same is true for $\operatorname{link}_{\Delta}(x)$ for any $x$. Since $\Delta$ has no homology in its top dimension, it follows that $\Delta$ cannot be semi-nonevasive.

Again related to the evasiveness conjecture, the proof of Theorem 5.10 implies that the deletion of the complex $\Delta_{n}^{2}$ with respect to any 0 -cell is nonevasive. An interesting but probably very hard problem is to characterize all monotone graph properties with this property.

It may also be worth mentioning that there exists a $\mathbb{Q}$-acyclic graph property that is not $\mathbb{Z}$-acyclic: Let $\Delta$ be the complex of all bipartite graphs on the vertex set $\{1,2,3,4,5,6\}$ that do not contain a subgraph isomorphic to the graph in Figure 5. Using the computer program homology [13], one may conclude that the only nonzero homology group is $\tilde{H}_{3}(\Delta ; \mathbb{Z}) \cong \mathbb{Z}_{2}^{16} \oplus \mathbb{Z}_{3}^{4} \oplus \mathbb{Z}_{9}$. We have not found a simple proof of this fact.

\section{Acknowledgment}

I thank Anders Björner, Svante Linusson, John Shareshian, Daniel Soll, and Volkmar Welker for interesting and fruitful discussions about topics considered in this paper. I also thank an anonymous referee for several useful comments and suggestions.

\section{References}

[1] C. A. Athanasiadis, Decompositions and connectivity of matching and chessboard complexes, Discrete Comput. Geom. 31 (2004), no. 3, 395-403. 
[2] E. Babson, A. Björner, S. Linusson, J. Shareshian, and V. Welker, Complexes of not $i$-connected graphs, Topology 38 (1999), no. 2, 271-299.

[3] A. Björner, Topological methods, Handbook of Combinatorics, R. Graham, M. Grötschel, and L. Lovász (eds), North-Holland/Elsevier, Amsterdam, 1995, 1819-1872.

[4] A. Björner, L. Lovász, S.T. Vrećica, and R.T. Živaljević, Chessboard complexes and matching complexes, J. London Math. Soc. (2) 49 (1994), 25-39.

[5] A. Björner and V. Welker, Complexes of directed graphs, SIAM J. Discrete Math. 12 (4) (1999), 413-424.

[6] A. Björner and M. Wachs, Shellable nonpure complexes and posets. I, Trans. Amer. Math. Soc. 348 (1996), 1299-1327.

[7] A. Björner and M. Wachs, Shellable nonpure complexes and posets. II, Trans. Amer. Math. Soc. 349 (1997), 3945-3975.

[8] B. Bollobás, Extremal graph theory, Handbook of Combinatorics, R. Graham, M. Grötschel, and L. Lovász (eds), North-Holland/Elsevier, Amsterdam, 1995, 1231-1292.

[9] S. Bouc, Homologie de certains ensembles de 2-sous-groupes des groupes symétriques, J. Algebra 150 (1992), 187-205.

[10] A. Chakrabarti, S. Khot, and Y. Shi, Evasiveness of subgraph containment and related properties, SIAM J. Comput. 31 (2001), no. 3, 866-875.

[11] H. Charalambous, Pointed simplicial complexes. Illinois J. Math. 41 (1997), no. 1, 1-9.

[12] M. K. Chari, On discrete Morse functions and combinatorial decompositions, Discrete Math. 217 (2000), 101-113.

[13] J.-G. Dumas, F. Heckenbach, B. D. Saunders, and V. Welker, Simplicial Homology, a share package for GAP, manual, 2000.

[14] R. Forman, Morse theory for cell complexes, Adv. Math. 134 (1998), 90-145.

[15] R. Forman, Morse theory and evasiveness, Combinatorica 20 (2000), 489-504.

[16] M. Hachimori, Combinatorics of Constructible Complexes, Doctoral thesis, University of Tokyo, 2000.

[17] P. Hersh, On optimizing discrete Morse functions, submitted.

[18] P. Hersh and V. Welker, Gröbner basis degree bounds on $\operatorname{Tor}_{\bullet}^{k[\Lambda]}(k, k) \bullet$ and Discrete Morse Theory for posets, Proceedings of the Summer Research Conference on Integer Points in Polyhedra, Snowbird, Utah, 2003.

[19] M. Hochster, Rings of invariants of tori, Cohen-Macaulay rings generated by monomials, and polytopes, Ann. of Math. (2) 96 (1972), 318-337.

[20] J. Jonsson. On the topology of simplicial complexes related to 3-connected and Hamiltonian graphs, J. Combin. Theory Ser. A 104, (2003), no. 1, 169-199.

[21] J. Kahn, M. Saks, and D. Sturtevant, A topological approach to evasiveness, Combinatorica 4 (1984), 297-306.

[22] D. J. Kleitman and D. J. Kwiatkowski, Further results on the Aanderaa-Rosenberg conjecture, J. Combin. Theory Ser. B $\mathbf{2 8}$ (1980), 85-95.

[23] T. Lewiner, H. Lopes, G. Tavares, Visualizing Forman's discrete vector field, Mathematical Visualization III, H.-C. Hege, K. Polthier (eds), Springer-Verlag, Heidelberg, 2002, 95-112. 
[24] T. Lewiner, H. Lopes, G. Tavares, Optimal discrete Morse functions for 2-manifolds, Comput. Geom. 26 (2003), no. 3, 221-233.

[25] T. Lewiner, H. Lopes, G. Tavares, Towards optimality in discrete Morse theory, Experiment. Math. 12 (2003), no. 3, 271-285.

[26] K. Mischaikow, M. Mrozek, and P. Pilarczyk, Graph approach to the computation of the homology of continuous maps, in preparation.

[27] Moriyama and Takeuchi, Incremental construction properties in dimension two - shellability, extendable shellability and vertex decomposability, Proc. 12th Canadian conference on computational geometry, Fredericton, 2000, 65-72.

[28] J. R. Munkres, Elements of Algebraic Topology, Menlo Park, CA, Addison-Wesley, 1984.

[29] J.G. Oxley, Matroid Theory, Oxford Univ. Press, 1992.

[30] J. S. Provan and L. J. Billera, Decompositions of simplicial complexes related to diameters of convex polyhedra, Math. Oper. Res. 5 (1980), 576-594.

[31] R. L. Rivest and J. Vuillemin, A generalization and proof of the Aanderaa-Rosenberg conjecture, 7th Annual ACM Symposium on Theory of Computing, Albuquerque, New Mexico, 1975, 6-12.

[32] R. L. Rivest and J. Vuillemin, On recognizing graph properties from adjacency matrices, Theoret. Comput. Sci. 3 (1976), no 3, 371-384.

[33] J. Shareshian, Discrete Morse theory for complexes of 2-connected graphs, Topology 40 (2001), no. $4,681-701$.

[34] J. Shareshian and M. L. Wachs, Torsion in the matching complex and chessboard complex, preprint.

[35] D. Soll, Evasive Simpliziale Komplexe und Diskrete Morse Theorie, Diploma Thesis, PhilippsUniversität Marburg, 2002.

[36] R. P. Stanley, Combinatorics and Commutative Algebra, second edition, Progress in Mathematics, vol. 41, Birkhäuser, Boston/Basel/Stuttgart, 1996.

[37] V. Turchin, Homologies of complexes of doubly connected graphs, Russian Math. Surveys (Uspekhi Mat. Nauk) 52 (1997), 426-427.

[38] V. Welker, Constructions preserving evasiveness and collapsibility, Discrete Math. 207 (1999) 243255.

[39] D.J.A. Welsh, Matroid Theory, Academic Press, 1976. 